$$
\text { D. }
$$




\title{
A first sketch of Computer Aided Ideation Exploring CAD tools as externalization media in design ideation
}

\author{
Philip Ekströmer
}

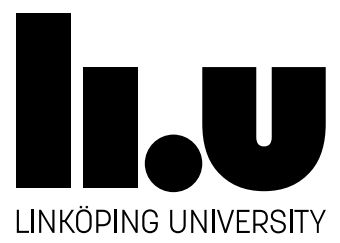

Division of Machine Design

Department of Management and Engineering Linköping University, SE-581 83 Linköping, Sweden

Linköping 2019 
A first sketch of Computer Aided Ideation. Exploring CAD tools as externalization media in design ideation

(C) Philip Ekströmer, 2019

Thesis no. 1859

ISSN 0280-7971

ISBN 978-91-7929-949-1

\section{Distributed by:}

Division of Machine Design

Department of Management and Engineering

Linköping University

SE-581 83 Linköping, Sweden

\section{Fonts}

Korolev (headings)

Miller (body, footnotes)

All illustrations by Philip Ekströmer

Images by Philip Ekströmer and Patrik Andersson

Printed in Sweden by LiU-Tryck, Linköping 2019 
Science is standing on the shoulders of giants. Someone might be standing on yours!

Johan Ölvander 



\section{ABSTRACT}

Even though Computer Aided Design (CAD) tools have changed the way designers work in most parts of the design process, designers still mostly use pen-and-paper sketching when generating design ideas. Previous studies exploring the use of CAD tools for design ideation have concluded that the tools available at the time did not support reflective conversation, serendipitous interpretation and creativity, making them unsuited for design ideation. However, many of these studies used tools now considered obsolete, implying that the conclusions might no longer be valid. With the variety and capabilities of current CAD tools, there is an opportunity for a new exploration of CAD tools in design ideation.

The aim of this licentiate thesis was to explore the use of CAD tools as externalization media in design ideation, what effect this has on the ideation process and how CAD tools might support design ideation. To this end, the thesis explored the use of CAD tools in design ideation in four studies. The first study consisted of a literature review on the strengths and weaknesses of sketches and CAD tools and a focus group discussion with three design experts. The second study compared master theses to explore how design representations used in the design process affect the breadth of design space exploration. The third study was a case study with two cases featuring the use of game engines and Virtual Reality for automotive lighting design and the fourth study compared the workflow in VR-sketching and penand-paper sketching.

The results of the studies in this thesis suggest that the notion that $\mathrm{CAD}$ tools are not useful for design ideation is no longer true. Based on expert evaluations and case studies, this thesis concludes that there are several opportunities for the use of CAD tools in design ideation. This is certainly true in design fields where it is difficult to make sketches. The potential strengths of using CAD tools for design ideation includes the ability to design in full scale and the ability to 
perform instantaneous transform operations, such as scaling and deforming. However, the ability to instantly undo in CAD tools has been identified as both a potential strength and potential a weakness for design ideation. While being able to rapidly undo mistakes could be beneficial to the ideation process, achieving a faster workflow with less time redoing and more time working on creating, this might also result in fewer opportunities for reinterpretation.

The conclusions in this thesis provide arguments for the use of CAD tools in design ideation, which could lead to new ways of generating, working with and thinking about design ideas. The findings also act as a stepping stone for further studies in the area of Computer Aided Ideation. 


\section{APPENDED PUBLICATIONS}

These four papers constitute the research foundation for this licentiate thesis. They are appended in full at the end of the thesis. The four papers will be referred to as Paper A-D in the text. The co-authors listed are the students who did the work which the cases are based on (Patrik Andersson, Johan Jönsson and Jens Wängdahl) and the main supervisor of the thesis, Renee Wever.

PAPER A

Ekströmer, P., \& Wever, R. (2019). “Ah, I see what you didn't mean” Exploring Computer Aided Design tools for design ideation. The Design Journal, 22 (sup1), 1883-1897.

\section{PAPER B}

Ekströmer, P., \& Wever, R. (2018). In search of lost materiality; The case of digital modelling in Industrial Design Engineering. DS 91: Proceedings of NordDesign 2018, Linköping, Sweden, 14th-17th August 2018.

\section{PAPER C}

Ekströmer, P., Wever, R., Andersson, P., \& Jönsson, J. (2019, July). Shedding light on game engines and virtual reality for design ideation. In Proceedings of the Design Society: International Conference on Engineering Design (Vol. 1, No. 1, pp. 2003-2010). Cambridge University Press.

\section{PAPER D}

Ekströmer, P., Wängdahl, J \& Wever, R. (2018). Virtual reality sketching. Unpublished paper based on working paper submitted to the 17th NORDCODE Seminar \& Workshop, Lund, 7-9 November, 2018. 



\section{ADDITIONAL PUBLICATIONS}

The following publications were published during the course of the research process, but are not included or discussed in the thesis.

Ekströmer, P., Nåbo, M., Pavlasevic, V., Eklöf, D., \& Wever, R. (2017). Challenging briefs; learning through client and designer roles in freshmen design education. In 19th International Conference on Engineering and Product Design Education (E\&PDE) (pp. 400-404). Design soc.

Ekströmer, P. Virtual reality sketching. Working paper submitted to the 16th Nordcode Seminar \& Workshop, Kalmar, 7-9 November, 2017.

Vistisen, P., Luciani, D., \& Ekströmer, P. (2019). Sketching Immersive Information Spaces: Lessons learned from experiments in 'sketching for and through virtual reality'. In 7th eCAADe Regional International Symposium Education and research in Computer Aided Architectural Design in Europe. eCAADe. 



\section{SHOUT OUT}

Theses generally have a section where they acknowledge people who have been of importance to their thesis. This is that section. However, I chose to call this section Shout out as an homage to rap battle culture, of which I'm a fan, where it is common to begin your round by giving a public acknowledgement, a "shout out", to sponsors or other people you want to mention. Right, here I go!

Shout out to the division of Machine Design. We have such a nice work atmosphere with great energy, lots of awesome ideas, great laughs and hands down the greatest fika discussions of all time! Big ups! Mini extra shout out to Team Machine who together rode Vätternrundan 2018. It was an epic (and long!) journey!

Shout out to my office colleagues, the Four Horsemen of Awesome, Malin Wilhelmsson, Ludwig Salén, Wanjun "Papa" Chu and Juan Felipe Muños Ruiz for a fun and creative office environment. Also, shout out to regular office visitor and dart champion David Beuger for being a cool dude and for lots of fun chats.

Shout out to Prof. Renee Wever for seeing a PhD in me, being a great and relaxed supervisor, having a great sense of humour and quality taste in pop culture and gifs (hard G) and not being too hard on me for not finding another design related supervisor. Also, shout out to him for being a master titlesmith and making puns that turn into thesis titles.

Shout out to Prof. Johan Ölvander for believing in me enough to hire me as a research assistant. Also, shout out to him for being my secondary supervisor and delivering deep quotes as feedback on this thesis. 
Shout out to Patrik Andersson, Johan Jönsson and Jens Wängdahl for making high quality master theses that I could use as cases. You guys did the hard work and deserve credit! Extra shout out to Jens for agreeing to try out VR sketching. It was a really useful first step towards further studies in the area.

Shout out to Mats Nåbo, Torbjörn Andersson and David Beuger for sharing your expert thoughts on the use of CAD tools in design ideation.

Shout out to Torbjörn Andersson, Fredrik Henriksson and Maral Babapour for writing quality licentiate theses that I could take inspiration from. Extra shout out to Torbjörn for making a InDesign template that I could use.

Shout out to Spotify's algorithm and their playlist curators for supplying me with a steady stream of Fantasy power metal songs.

Last, but certainly not least, shout out to my family for your love and support, to my pets Nike and Minerva for quality cuddles and to Emelie for being amazing and supporting me when I didn't feel like going on.

Philip Ekströmer, November 2019 
Page intentionally left blank 


\section{CONTENTS}

INTRODUCTION

1.1. Delimitations ..................................... 2

1.2. Thesis outline .......................................

FRAME OF REFERENCE

2.1. Design ideation. ................................. 5

2.2. Externalization .................................. 7

2.3. CAD tools in design ideation........................... 11

2.4. Implications for the thesis............................ 14

RESEARCH METHODOLOGY 17

3.1. Background and empirical context................... 17

3.2. Design research and research design ................ 18

SUMMARY OF PAPERS

4.1. Exploring CAD tools for design ideation ............... 25

4.2. In search of lost materiality ...................... 27

4.3. Shedding light on game engines and virtual reality for automotive lighting design........................... 28

4.4. Virtual reality sketching .......................... 29

OUTCOMES 33

5.1. What potential strengths do CAD tools have when employed for design ideation?................................. 33

5.2. What are the effects of using CAD tools for design ideation? 
DISCUSSION \& IMPLICATIONS 39

6.1. Undo or undon't ................................... 39

6.2. Full scale designing ............................. 40

6.3. Workflow ..................................... 41

6.4. Single tool process ............................. 41

6.5. Additions to literature .......................... 42

6.6. Reflections on limitations............................ 43

CONCLUSIONS \& FURTHER STUDIES

7.1. Tentative future work ........................... 48

7.2. Further studies ............................... 49

REFERENCES 


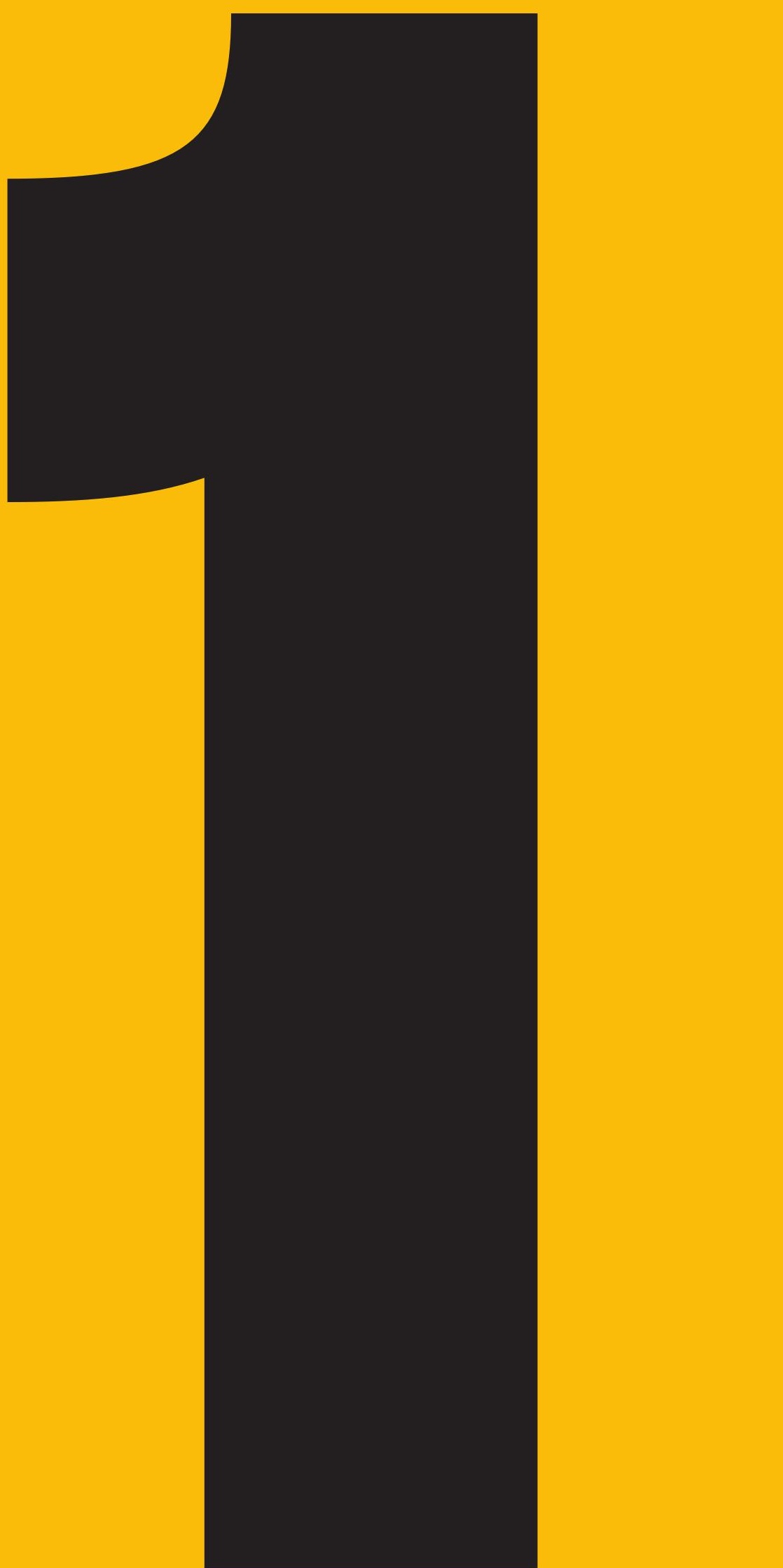




\section{D) INTRODUCTION}

Using a tool, be it fingers, sticks or rocks to make a mark on some kind of medium has been a way to show and store thoughts for most of human history. With the introduction of affordable paper during the Renaissance (Thomas Buser, n.d.), artists, painters and sculptors were able to rapidly visualize possible futures in a cheap and disposable way. Today, the masters of the Renaissance have been replaced by designers and architects and most parts of the design process are aided by computers. However, design ideation, the generation of design ideas, is still mostly pen-and-paper based. While there have been several studies exploring the use of Computer Aided Design (CAD) tools for design ideation in the last two decades (e.g. Stappers and Hennessey (1999), Charlesworth (2007) and Alcaide-Marzal et al. (2013)), many have found that the available CAD tools at the time did not support reflective conversation, serendipitous interpretation ${ }^{\mathrm{a}}$ and creativity, making them unsuited for design ideation. However, as the capabilities of CAD tools have increased since, the conclusions from studies undertaken with tools now considered obsolete might no longer be valid. With the variety and capabilities of current CAD tools, there is an opportunity for a new exploration of CAD tools in design ideation.

\section{AIM AND RESEARCH QUESTIONS}

This thesis aims to explore the use of CAD tools as externalization media in design ideation, what effect this has on the ideation process and how CAD tools might support design ideation. To address this aim, the following two research questions were posed:

a. This is also known as "happy accidents". The painter Bob Ross often said "We don't make mistakes; we just have happy accidents”. 
RQ 1 What potential strengths do CAD tools have when employed for design ideation?

RQ 2 What are the effects of using CAD tools for design ideation?

\subsection{DELIMITATIONS}

This licentiate thesis explores the use of CAD tools as externalization media in the creation of physical artefacts (i.e. products). Thus, the tools presented in the thesis are generally related to the practices of product design, industrial design and industrial design engineering. Consequently, this thesis studies the activities of designers and design engineers. Moreover, while design ideation can be both an individual and a team activity, the focus of this thesis is how individual ideation is supported by the interaction with an externalization tool.

Further, there is a need to clarify what is meant by CAD, as the term has different meanings depending on context. As the acronym CAD stands for Computer Aided Design ${ }^{\mathrm{b}}$ it may include any tool that uses computers to aid design, from digital creative prompt tools (e.g. Design(human)design by Mothersill \& Bove Jr (2017)) to software suites supporting multiple stages of the design process. However, this thesis uses the term CAD tools to mean passive, human controlled, mark-making computer tools. Thus, the term CAD tools in this thesis is used for computer tools that can be used as an extension of the human mind, allowing it to externalize and visualise ideas.

However, the available CAD tools that match this definition have very different uses and application areas. Therefore, this thesis focuses on the subset 3D CAD tools, tools used to create artefacts in threedimensional (3D) space.

b. The acronym may also stand for Computer Aided Drafting (Madsen \& Madsen, 2016). Outside engineering and design, it may be known as the currency code for Canadian dollars. 


\subsection{THESIS OUTLINE}

This thesis is comprised of seven chapters. Below is an outline of the contents of the following chapters.

\section{CHAPTER 2 FRAME OF REFERENCE}

This chapter introduces literature on design ideation, externalization, sketches and sketching in design, and the use of CAD tools in design ideation. The aim of this chapter is to provide an overview of the literature and position the thesis in relation to previous and current research.

\section{CHAPTER 3 RESEARCH METHODOLOGY}

This chapter presents the research approach, including theoretical and methodological considerations as well as the author's background and epistemological stance.

\section{CHAPTER 4 SUMMARY OF PAPERS}

This chapter presents a summary of each of the four appended papers (A-D).

\section{CHAPTER 5 OUTCOMES}

This chapter presents the findings from the four studies.

\section{CHAPTER 6 DISCUSSION AND IMPLICATIONS}

This chapter discusses the findings and the implications of using CAD tools for design ideation and design in general.

\section{CHAPTER 7 CONCLUSIONS AND FURTHER STUDIES}

The last chapter presents the answers to the research questions and the overall conclusion that CAD tools can have a use in design ideation. Lastly, it details the direction for future studies in the area of Computer Aided Ideation. 


\section{D) FRAME OF REFERENCE}

The focus of this thesis is designers use of $3 \mathrm{D}$ CAD tools as externalization media in design ideation. This can be visualized as a Venn-diagram, where the intersection between the areas externalization, design ideation and CAD tools provides the focus area for the thesis. This is pictured in Figure 1. This chapter provides an overview of the three areas that form the Venn-diagram, with an emphasis on literature regarding the use of CAD tools for design ideation.

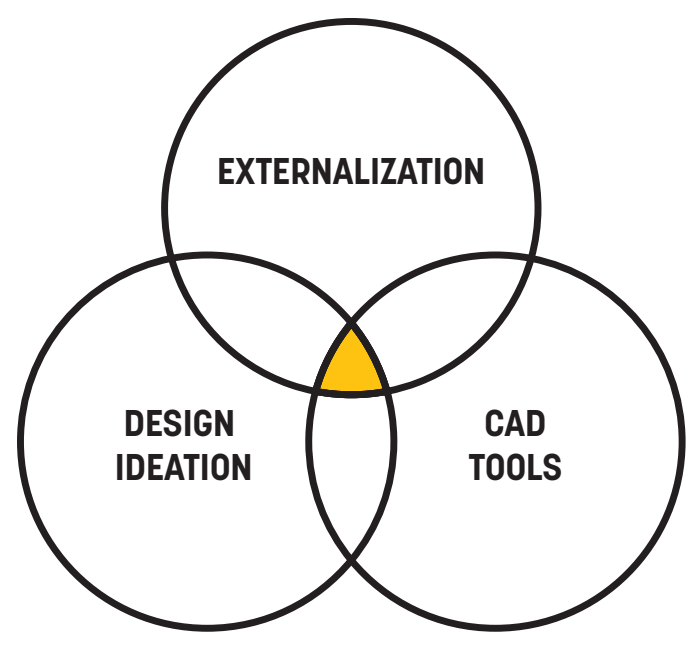

Figure 1 The intersection of areas that are of interest for this thesis

\subsection{DESIGN IDEATION}

Design has been a natural part of human life since we consciously started to change and improve our world and today almost everything we interact with has been designed in some way (Wikberg-Nilsson et al., 2015). In general, design can be described as a process of creation. 
Models of the design process usually divide the process into several stages or phases. A basic design process, shown in Figure 2, can be summarised in three steps: 1) define the problem space 2) generate possible solutions and 3) Evaluate and select the solution that adequately solves the problem (Boekholt as cited in Pei (2009)).

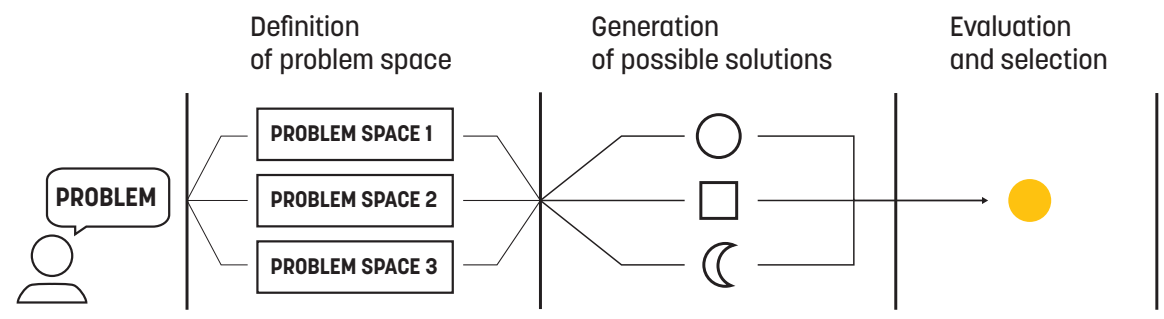

Figure 2 Boekholt's design process. Adapted from Vries [2019]

Depending on the model, the design process may have more stages (e.g. six stages in Ulrich and Eppinger (2008)) and different names for the stages. This thesis will a use model used for developing products with four stages as a reference point. The model is shown in Figure 3.

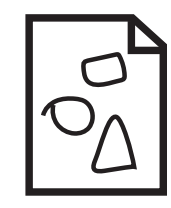

Concept design

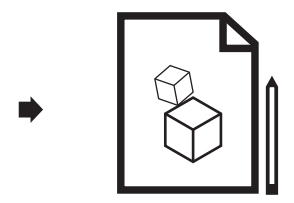

Concept development

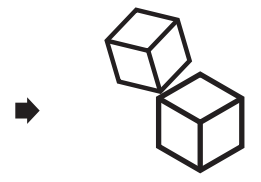

Embodiment design

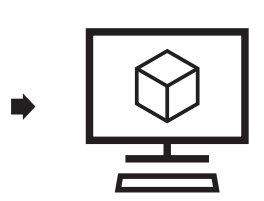

Detail design

Figure 3 The four stages of a design process for physical products. Adapted from Pei (2009] 
The first stage of this model is concerned with generating ideas, the second with developing, refining and evaluating ideas, the third with producing concrete representations of the developed idea and the fourth with making final and detailed technical descriptions of each component in the product (Pei, 2009). This thesis is focused on the first stage, which is where designers explore the design space, generate ideas and transfer them from their minds into the real world through some kind of medium. The activities in this stage are known as Ideation (e.g. Dorta, Pérez and Lesage (2008)), Design Ideation (Jonson, 2005) or Conceptualization (e.g. Bilda and Demirkan (2003)). This thesis will use the terms Design ideation and Ideation interchangeably. Jonson describes design ideation as "a matter of generating, developing and communicating ideas, where 'idea' is understood as a basic element of thought that can be either visual, concrete or abstract" (Jonson, 2005, p. 613).

\subsection{EXTERNALIZATION}

The ability to visualize thoughts comes natural to most humans ${ }^{\mathrm{a}}$. It is usually envisaged that the process of creating design ideas occurs in the "mind's eye" (Tovey et al., 2003), a conceptual "organ" representing the complex processes in the brain that allow us to create representations of objects or people that are not in the field of view (Ferguson, 1994). However, to be able to develop the idea into a product, it needs to be transferred from the mind's eye into the real world through a medium. In design, this act is called externalization. One of the reasons for designers to externalize their thoughts is that words, pictures or models can radically increase the mind's ability to visualize (Fish and Scrivener, 2007). This is discussed further in the section on sketching. Another reason for using externalizations is to lighten the load on their short-term memory. In classical problem solving (e.g. Simon (1970)), externalizations are used when the complexity and/or size

\footnotetext{
a. With the exception of those who have Aphantasia, a condition where you cannot bring an
} image to mind (Clemens, 2018) 
of a problem exceeds the limits of the short-term memory, which is where problem solving takes place. As design problems by nature are ill-structured ${ }^{\mathrm{b}}$ (Simon, 1973), implying that they are not fully defined and have many possible solutions, they are very likely to exceed the limit of the short term memory. There is however a study by Bilda et al. (2006), showing that expert designers can produce solutions of similar quality without externalizing ${ }^{\mathrm{c}}$.

\section{SKETCHING AND SKETCHES}

In design, the use of sketching to externalize ideas has become so ubiquitous that the process of sketching is almost impossible to separate from designing in general and ideation in particular. During their education, designers, architects and other design related professions are taught to use sketching to explore the design space, develop ideas and trigger new ideas (Bilda et al., 2006; Do, 2005), making sketching the natural way to think about and solve design problems. This section will briefly define the terms sketching (the act/ process) and sketches (the result/media) and discuss how they are important for design ideation.

Principally, sketching is a process of making quick freehand drawings. However, in design, sketching is not used to depict anything that already exists; rather it is used to externalize what the designer has seen in her mind's eye (Tovey et al., 2003). Sketching is also used to probe design problems. Schön (1991) describes this as a dialectic process, a conversation between the designer and the sketch, where the designer makes a mark on the paper and the sketch talks back to her, initiating a reflective loop (Figure 4). This conversation is possible thanks to the ambiguous nature of sketches, which is perhaps the most prominent value of sketches in regard to ideation (Buxton,

b. Also "ill-defined" or "underdetermined" (Dorst, 2003)

c. As a parallel example, consider the wizarding wands in the world of Harry Potter. Wands are used to centralize the magic effects for more complex results. While spells can be cast without the wand, wandless magic is very difficult and requires much concentration and incredible skill ("Wizarding world", 2017). The same goes for ideating without externalization. You can keep it all in your head, but it requires much concentration and skill. 
2007; Goel, 1995; Goldschmidt, 1991; Schön, 1991). The ambiguity of sketches allows the designer to interpret her own or someone else's sketch as something different than what was first intended, thereby setting the ideation on a new path (Fish and Scrivener, 2007). This iterative cycle of ideas, sketches, reinterpretations and new ideas helps to reduce the ill-defined nature of design problems (Purcell and Gero, 1998).

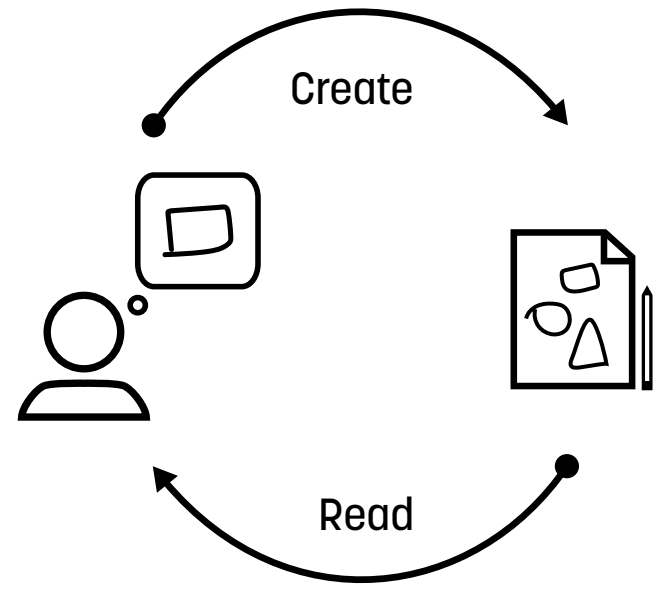

Figure 4 The "conversation" between the mind and the sketch. Adapted from Buxton (2007)

Sketches may be described as "representations used to assist in the visualisation and mental manipulation of some unfinished or imaginary object" (Fish, 1996, p. 25). Sketches have several different functions in design, such as thinking sketches (Ferguson, 1994), used to support the designers individual thinking process, talking sketches (Ferguson, 1994), used to support a group discussion, prescriptive sketches (Ferguson, 1994), used to communicate design decisions to persons outside the design process and storing sketches (van Der Lugt, 2005) used to archive design ideas for future reference. There are several benefits of using sketches in design ideation. The material 
cost of pen and paper is low compared to other tools (Jonson, 2002) and sketches can be made using scrap paper and any item that leaves a mark, making it easy to capture an impulsive idea (Lim et al., 2004). A sketch can be transformed by drawing over parts of it, by adding to it or by erasing (Goldschmidt, 1991; Jonson, 2002; Pour Rahimian et al., 2012). Furthermore, the simple interface allows for fast expression (Lim et al., 2004) and gives the designer the ability to make many alternatives in a short period of time. Moreover, there are very few disadvantages of sketches in design ideation. However, sketches are two-dimensional representations of 3D objects (Sener et al., 2002), meaning that the use of a sketch to depict a $3 \mathrm{D}$ object involves a loss of dimensionality. Also, the analogue nature of sketches makes it difficult to transition to tools being used in later stages of the design process (Pour Rahimian et al., 2012).

Sketching in general and idea sketching in particular is, with few exceptions, studied byusing protocolanalysis(Goel,1995; Goldschmidt, 1991; Schön, 1991; Suwa and Tversky, 1997). This method originates from psychology research and is used for controlled observation and experimental analysis of cognitive behaviour (Ericsson and Simon, 1984). Using design protocols provides the researchers with rich material and insights into the designer's mind. There are in general two ways of conducting a protocol analysis: concurrent (also known as talk out loud) and retrospective verbalization (Gero and Tang, 2001). In concurrent verbalization, the designers are asked to express what they are thinking as they are doing it, which gives the researchers direct access to the designer's thought process. However, talking while designing may hinder the creative process as it mostly relies on visual imagery, which cannot be expressed using verbalization (Verstijnen et al., 1998). When using retrospective verbalization, the design activity is video recorded and, when the design task is finished, the designers explain their thinking process while looking at the recording. This allows the designer to focus completely on the design task. However, it can be hard to remember thought processes after completing the task, which may prompt the designer to report false memories to give an impression of rational behaviour (Newell, as cited in Shih et al. (2017)). 


\subsection{CAD TOOLS IN DESIGN IDEATION}

The era of CAD tools in design is often said have begun when Ivan Sutherland (1968) created SKETCHPAD ${ }^{d}$, the first graphical interface for computers, allowing CAD tools to become an externalization media useful for design. However, while CAD tools are essential in most parts of the design process today, designers still mostly use pen-and-paper sketching in design ideation. Because this is the case, several studies have investigated the nature of sketching in design ideation (e.g. Verstijnen et al. (1998), Do (2005) and Babapour (2015)) to develop CAD tools that better support designers' ideation processes, using the knowledge gained from studying sketching. Researchers have argued that if CAD tools are to compete with sketching, they must be intuitive, allow ambiguous representations and effortless exploration of ideas and be able to capture sudden thoughts and ideas (Alcaide-Marzal et al., 2013; Hacker et al., 2002; Pei et al., 2009; Séquin, 2004; Verstijnen et al., 1998). However, when exploring the use of available CAD tools, many studies have found that CAD tools have limited to no use in design ideation. Early studies concluded that CAD tools of the time did not live up to the requirements of design ideation and were only useful for the later stages of the design process (Stappers and Hennessey, 1999; Verstijnen et al., 1998). Later studies have found that pen-and-paper sketching is better for generating solutions (Alcaide-Marzal et al., 2013; Stones and Cassidy, 2007), reinterpretation (Alcaide-Marzal etal., 2013; Stones and Cassidy, 2010) and conceiving the problem (Bilda and Demirkan, 2003). One of the major reasons CAD tools are deemed unsuited for ideation is that they are argued to not support ambiguous representations (van Dijk, 1995; Hennessey and Stappers, 1999; Verstijnen et al., 1998) and therefore cannot enable the conversation with the material that allows the designer to see new directions for her original thought (Charlesworth,

d. Sutherland, with the help of student Bob Sproull, also created what is considered the first Virtual Reality Head Mounted Display systems. The device was so heavy it had to be suspended from the ceiling, which gave it the name Sword of Damocles. This is a reference to the legend about Damocles who was offered to switch place with king Dionysius for a day, but had to sit on a throne above which a huge sword was held by a single horse hair. 
2007). Moreover, it has been suggested that designers find their ideas and thinking constrained by the functional capabilities of the CAD tool, which limits creativity (Chester, 2007; Kosmadoudi et al., 2013; Robertson and Radcliffe, 2009). In one of the more recent studies, Alcaide-Marzal et al. (2013) concluded that CAD tools, although far more flexible than their predecessors, are still too restrictive in terms of creativity.

There are, on the other hand, studies that argue for the use of CAD tools in design ideation. van Dijk (1995) concluded that it should be feasible to use CAD tools in ideation after exploring a prototype CAD tool for design ideation with eight designers. Similarly, Stappers and Hennessey (1999) suggested that CAD tools would be useful for ideation within a foreseeable future. In other studies it has been argued that using a CAD tool elicits a more complex cognitive behaviour and more frequent reinterpretation when compared to pen-and-paper sketching (Won, 2001) and that CAD tools can help design cognition, creativity and intuition (Hanna and Barber, 2001). Jonson (2005), one of the first to mention Computer Aided Ideation, argued that commercial CAD tools tend to be driven by efficiency and accuracy rather than creativity and that this might lead to overlooking the ways of perceiving and conceiving design that CAD tools can provide. Alcaide-Marzal et al. (2013) reflect that the problem might not only be the limitations of the CAD tools but also how designers have to work with them. Moreover, they argue that the feeling of traditional sketching seems to be very difficult to replace and suggest using CAD tools as a second concept generation stage after rough sketches (Alcaide-Marzal et al., 2013).

CAD tools for design ideation are commonly evaluated by comparing them to pen-and-paper sketches. A common method that yields an easily comparable result is to conduct a statistical analysis of the outcomes, such as number of sketches or reinterpretations (AlcaideMarzal et al., 2013; Oh et al., 2005). Several studies also analyse the artefacts from the different media and use this as the point of 
comparison (Alcaide-Marzal et al., 2013; Knight et al., 2005; Won, 2001). Other less common ways to compare is to use self-assessment (Dorta et al., 2009), expert ratings (Oh et al., 2005) or design diaries (Charlesworth, 2007). The benefits of a comparison is that it is possible to conclude which tool is the better one. However, as with all controlled experiments, the experiment procedure produces an unnatural environment that is not representative for how ideation is done in the real world.

To capture how CAD tools are used in the real world, researchers have employed case studies (Hjelseth et al., 2015; Jonson, 2005; Robertson and Radcliffe, 2009) and observations (Pour Rahimian et al., 2012). The conclusions from these studies are innately less rigid and generalizable. However, some of the most interesting insights about CAD tools for design ideation have come from case studies. In a case study of a small engineering team, Robertson and Radcliffe (2009) found that using CAD tools enhanced the visualization and communication, but also that ideas and thinking can be constrained by the functional capabilities of the CAD tool (circumscribed thinking) and that $\mathrm{CAD}$ tools can cause premature fixation and bounded ideation. In a later survey of CAD users they found that premature fixation, circumscribed thinking and enhanced visualization and communication were quite widespread in engineering design practice, while bounded ideation occurred relatively infrequently (Robertson and Radcliffe, 2009). Hjelseth et al. (2015) used a combined approach of case studies and research through design to explore the use of game engines as a design tool to conceptualise and simulate user scenarios in the maritime domain. An interesting conclusion from this study is that game engines have the ability to trigger reflective conversation.

Much like studies of sketches, there are several studies of CAD tools using protocol analysis. Bilda and Demirkan (2003) use protocol analysis to gain insight into the cognitive processes while using CAD tools and pen-and-paper sketching and Lee and Yan (2016) investigate how the interface of a CAD tool affects the ideation by comparing two 
CAD tools using protocol analysis. In a series of studies, Musta'amal, Norman and Hodgson (2009a, 2008, 2009b), use protocol analysis combined with several other qualitative methods to explore creative behaviours when using CAD tools.

\subsection{IMPLICATIONS FOR THE THESIS}

Many of the claims that CAD tools have no use for design ideation are based studies using tools that are now considered obsolete and the conclusions from these studies may no longer be true with the variety and capabilities of current tools. Moreover, the majority of the studies only capture the use of a single CAD tool. While this might once have been enough to assess the general status of CAD tools, the current variety of CAD tools make such generalizations debatable. Also, the comparisons between CAD tools and pen-and-paper sketching are generally focused on how the CAD tool differs from pen-and-paper sketching. Thus, they rarely discuss the potential CAD tools have to change the way design ideation is perceived and conceived (Jonson, 2005). In summary, this implies that is sensible to use an approach that provides several perspectives on the use of CAD tools for design ideation. The different perspectives can be acquired by exploring cases where CAD tools for ideation seem to make most sense and investigating the current practices of designers and design students. 



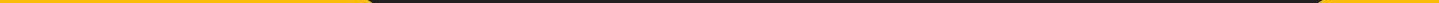




\section{D) RESEARCH METHODOLOGY}

For the reader to understand the meaning of the research questions and the quality of the findings, it is important to gain insight into the authors' epistemological stance, theoretical perspective and methodological approach. Thus, this is the section where I state my views on what constitutes knowledge and my process of acquiring it.

\subsection{BACKGROUND AND EMPIRICAL CONTEXT}

I am educated as an industrial design engineer, meaning I have been exposed to tools used in both industrial design and engineering practice. While emphasising the importance of sketching in design, my design educators also encouraged the use of CAD tools in ideation and concept development. Thus, during my education, I have explored several ways of externalizing design ideas. I also have experience of using (mostly 2D) CAD tools for ideation from freelance work as a graphic designer. While I acknowledge the usefulness of sketching in design ideation, I am making the argument that pen-and-paper sketching is just a tool among other tools for design ideation. I am also, to some extent, debating the current predominant view in literature that CAD tools cannot be used for design ideation.

Coming from an industrial design engineer background, my view on knowledge is influenced by both engineering, which has a basis in the natural sciences, and design which has its roots in both art and the social sciences. My basic assumption regarding knowledge is that it is both constructed and based on an external world that exists independently from our ideas about it. I take a pragmatist stance, which is not committed to one system of philosophy and reality (Creswell, 2003). In the pragmatist view, the problem is more important than methods, allowing a mixed method research approach where any available method may be used (Creswell, 2003). This implies that I, in my research, am comfortable using both qualitative methods, 
such as case studies and interviews, and quantitative methods, such as controlled experiments, and combinations thereof, to explore and investigate the use of $\mathrm{CAD}$ tools in design ideation.

\subsection{DESIGN RESEARCH AND RESEARCH DESIGN}

Stappers and Giaccardi (2017) define three major categories of design research (Figure 5): Research into design, research for design and research through design. Research into design means using research methods to study the process of designing. Research for design implies using research methods, e.g. observations and interviews, to learn specific information about the situation for which the design is made. This is commonly used in design practice. The last category, research through design, is quite different from the former two as it involves generating new knowledge by creating specific solutions, allowing the designers to make discoveries through their own design process (Stappers and Giaccardi, 2017). The research method in this thesis falls into the first category, research into design, as it aims to investigate the process of designing and explore how it could be done differently.

\section{RESEARCH}

INTO DESIGN
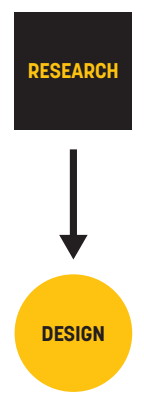

RESEARCH

FOR DESIGN
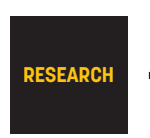

RESEARCH

THROUGH DESIGN

Figure 5 Three categories of design research. Adapted from Stappers and Giaccardi (2017) 
Based on the argumentation in section 2.6, comparing the use of a single CAD tool to pen-and-paper sketching is no longer a reasonable way to make claims about the status of CAD tools for design ideation. Therefore, this thesis aimed to explore the current state of CAD tools for design ideation by using multiple study methods and exploring several CAD tools. The overall research approach used in the thesis is a mixed methods approach, where qualitative and quantitative data is combined to provide an analysis of the research problem (c.f. concurrent procedures in Creswell (2003)). The research consisted of four separate studies, using different CAD tools and study methods, that together present several perspectives on the use of CAD tools for design ideation. Figure 6 shows the four studies and the respective research question(s) they answer and Figure 7 shows a timeline of the thesis work with studies undertaken on the top row, papers written in the middle row and conferences where the work has been presented on the bottom row. Below follows a summary of the research methods used in each study. A more extensive summary of the papers can be found in Chapter 4.

Study A consisted of 1) a literature review compiling the strengths and weaknesses of sketches and CAD tools for design ideation 2) an expert evaluation of four different CAD tools for design ideation using a Likert-scale survey based on the compiled strengths and weaknesses and 3) a focus group discussion (Van Boeijen et al., 2014; Hanington and Martin, 2012; Wikberg-Nilsson et al., 2015) with the experts that was analysed using thematic analysis (Braun and Clarke, 2006). By compiling the strengths and weaknesses of CAD tools reported in literature and collecting experts opinions on the use of CAD tools in design ideation, study A provided answers to both RQ 1 and RQ2.

Study B explored the relation between the design representations that are used in the design process and the breadth of design space exploration. To explore this, 25 finished master theses on product design and development were compared using the design representations used (sketches, sketch models, functional models, $\mathrm{CAD}$ or Other) and the diversity of ideas presented in the thesis as the 


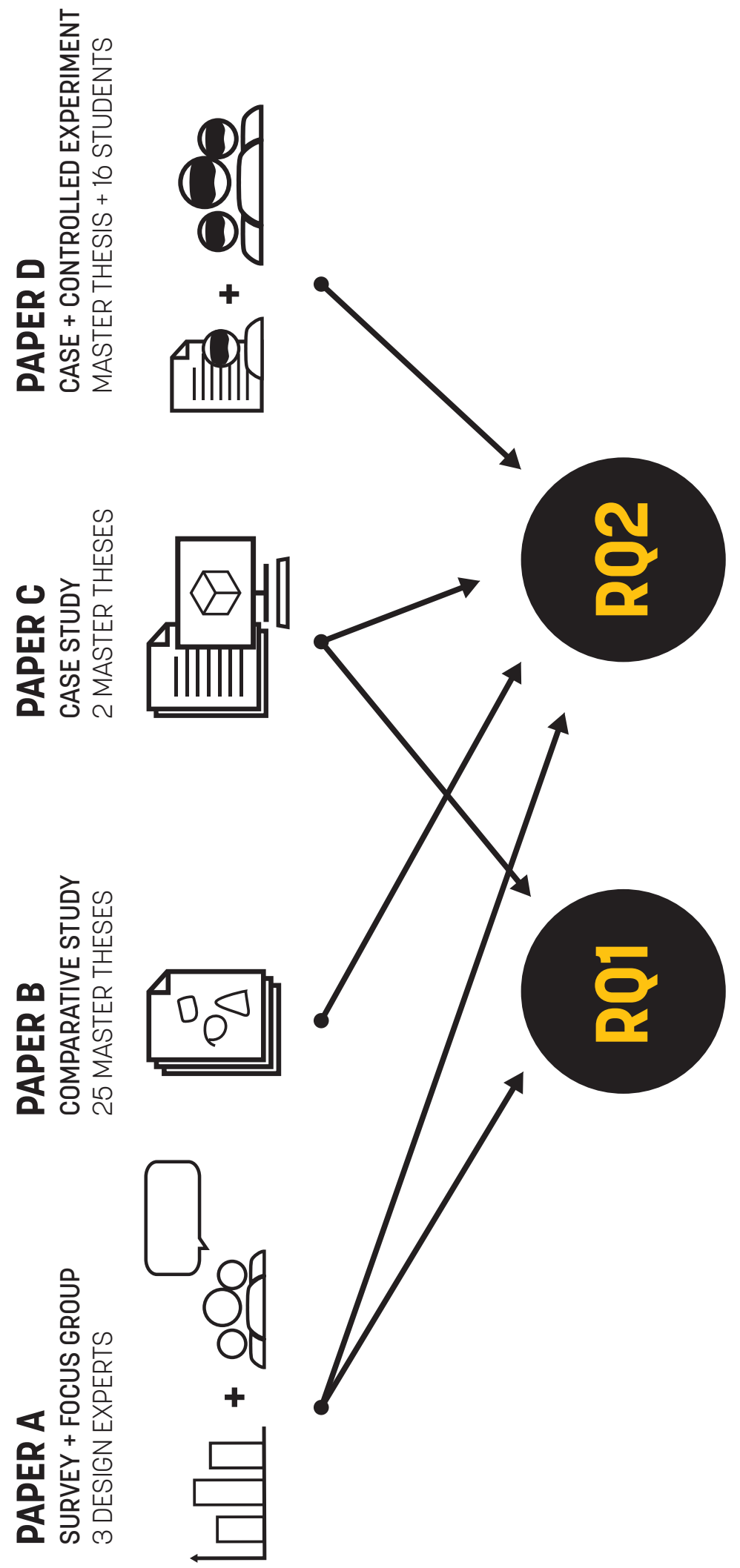

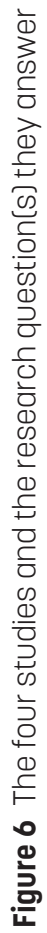




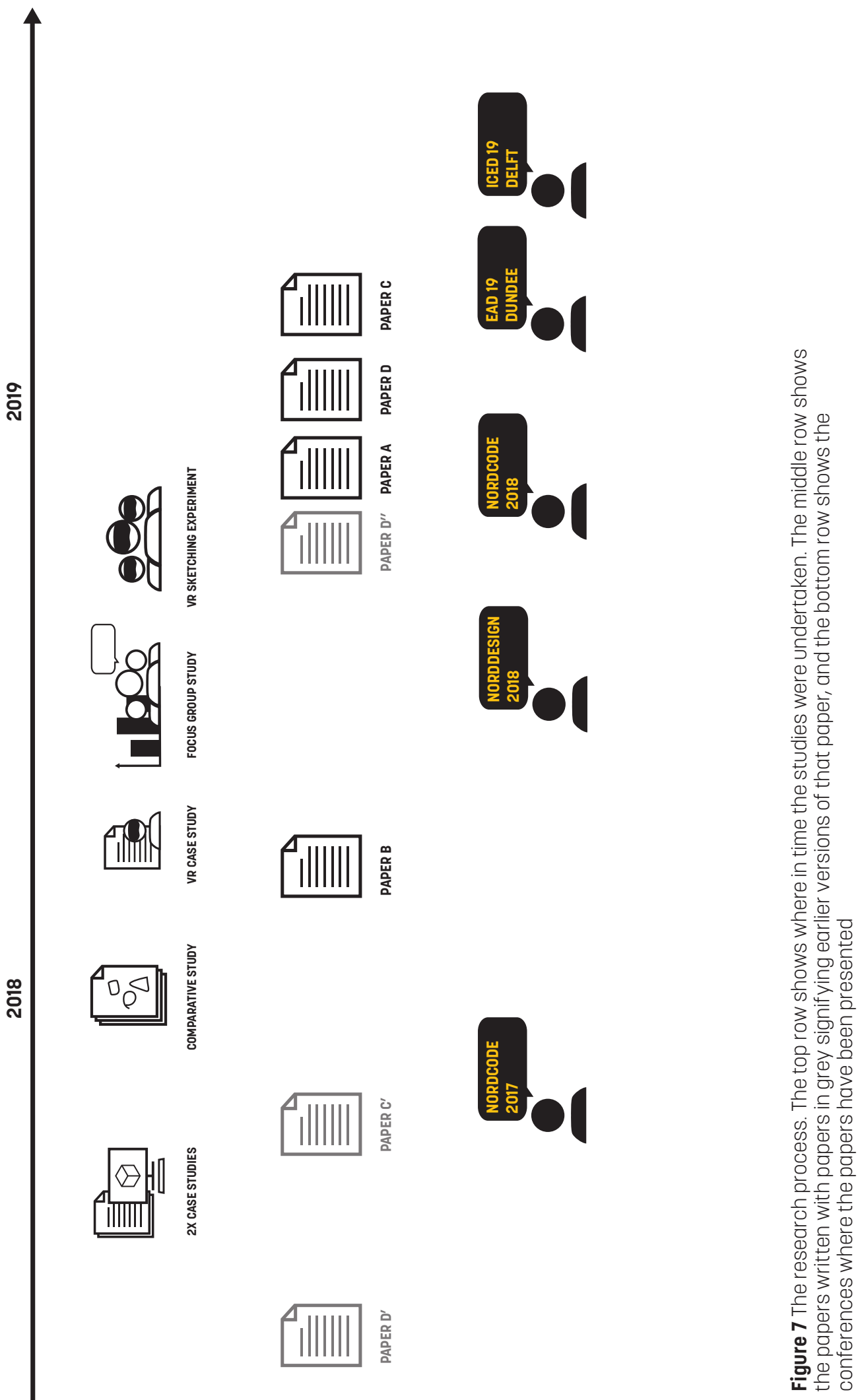


two parameters. Using several finished master theses allowed a wider sample of works as opposed to following a single master thesis work and documenting their process. The conclusions from this study were used to answer RQ 2.

Study C was a case study (c.f. Teegavarapu and Summers, (2008)), based on two master theses by Andersson (2017) and Jönsson (2017) respectively. Both explored using a game engine for design ideation in automotive lighting design. The cases were based on parts of the master theses featuring exploratory research with a research through design approach. The two cases, where a game engine and VR was used as an externalization media in a setting where sketching is not applicable, provided both answers to RQ 1 and RQ 2, as well as insights on how the benefits of these tools might be used in other areas of design where sketching can be used.

Lastly, Study D contained a case where Virtual Reality (VR) sketching was explored and a comparative experiment with 16 participants investigating the differences in workflow between VR-sketching and pen-and-paper sketching. This study provided insights that were used to answer RQ 2. 



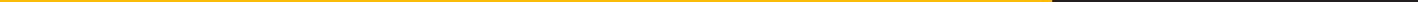




\section{D) SUMMARY OF PAPERS}

This chapter provides a summary of the appended papers A-D, including the rationale behind the studies, the methods used and the results that were obtained.

\subsection{EXPLORING CAD TOOLS FOR DESIGN IDEATION}

Paper A collected expert opinions on the use of CAD tools for design ideation by having three experts evaluate four CAD tools followed by a focus group discussion. It started from the proposal that for a CAD tool to be useful for design ideation, it should retain the strengths of sketches and add the strengths of CAD tool. To be able to evaluate CAD tools using these parameters, the strengths and weaknesses of sketches and CAD tools were first compiled through a literature review. The compiled strengths and weaknesses were then reviewed and expanded upon by the three experts and used in a Likert-scale survey where the experts rated four different CAD tools (SketchUp, Blender, Unreal Engine 4 and Fusion360). These tools were chosen as they represent a wide range of the capabilities of current CAD tools and because the experts were familiar with them. The experts also rated pen-and-paper sketches for comparison. To make the survey statements uniform, the strengths and weaknesses were formulated as beneficial characteristics for design ideation. This meant that the weaknesses of CAD tools and sketches were reformulated as benefits. The resulting beneficial characteristics for design ideation are shown in Table 1 . The ratings in the survey were weighted based on the experts' proficiency with the tools and used as a basis for a focus group discussion with the experts. The discussion was recorded, transcribed and analysed using thematic analysis. Based on this discussion, the paper suggests that the notion that CAD tools are not useful for design ideation is no longer true and that there are several opportunities for the use of CAD tools in design ideation. 
Table 1 List of beneficial characteristics for design ideation and their source. The source for respective characteristic is marked with an X in the table

\section{Beneficial characteristics for design ideation Literature Weaknesses Experts}

\begin{tabular}{|c|c|c|}
\hline $\begin{array}{l}\text { The tool supports new ways of } \\
\text { viewing the original thoughts }\end{array}$ & $x$ & \\
\hline The tool supports creativity & $x$ & \\
\hline The tool supports doodling & $x$ & \\
\hline The tool has an intuitive interface & $x$ & \\
\hline $\begin{array}{l}\text { Ideas and thinking are unconstrained } \\
\text { by the capabilities of the tool }\end{array}$ & $x$ & \\
\hline $\begin{array}{l}\text { The tool supports a three-dimensional } \\
\text { representation of a three-dimensional object }\end{array}$ & $x$ & \\
\hline $\begin{array}{l}\text { The tool supports ambiguous } \\
\text { representations }\end{array}$ & $x$ & \\
\hline The tool supports fast expression & $x$ & \\
\hline The tool is easy to learn and use & $x$ & \\
\hline The tool is low cost & $x$ & \\
\hline $\begin{array}{l}\text { The tool is flexible, it is easy to transform } \\
\text { and correct the representation }\end{array}$ & $x$ & \\
\hline The tool is almost always available & $x$ & \\
\hline The tool supports a co-design process & $x$ & \\
\hline $\begin{array}{l}\text { The tool supports multiple information } \\
\text { types other than illustrations, such } \\
\text { as words and material samples }\end{array}$ & & $x$ \\
\hline $\begin{array}{l}\text { The tool supports the creation } \\
\text { of representation that are not } \\
\text { visualizations of the object }\end{array}$ & & $x$ \\
\hline $\begin{array}{l}\text { The tool has a low threshold } \\
\text { for getting started }\end{array}$ & & $x$ \\
\hline $\begin{array}{l}\text { The tool supports easy transformation } \\
\text { (rotation, scaling, zooming and panning) }\end{array}$ & $x$ & \\
\hline
\end{tabular}




\begin{tabular}{|c|c|c|}
\hline $\begin{array}{l}\text { The tool supports temporally omitting } \\
\text { an object or group of objects }\end{array}$ & $x$ & \\
\hline $\begin{array}{l}\text { The tool facilitates the creation of } \\
\text { shapes that are hard to do by hand }\end{array}$ & $x$ & \\
\hline The tool has automatic undo and redo & $x$ & \\
\hline $\begin{array}{l}\text { The tool supports automatic repetition } \\
\text { and/or cloning and/or duplication }\end{array}$ & $x$ & \\
\hline $\begin{array}{l}\text { The tool supports accurate } \\
\text { representation of materials }\end{array}$ & & $x$ \\
\hline The tool supports Generative design & & $x$ \\
\hline The tool supports design in full scale [VR] & & $x$ \\
\hline $\begin{array}{l}\text { The tool supports visualizations of non- } \\
\text { physical concepts such as light and sound }\end{array}$ & $x$ & \\
\hline
\end{tabular}

\subsection{IN SEARCH OF LOST MATERIALITY}

Paper B explored the relation between the design representations used and the breadth of design space exploration. To explore this, 25 finished master theses from the division of Machine Design at LiU between 2008 and 2017 were compared using the design representations used (sketches, sketch models, functional models, CAD or Other) and the diversity of ideas presented in the thesis as the two parameters. The results were compared visually by making graphs where the design representations has different colours and the height of the graph depicts the diversity of the ideas. An example is shown in Figure 8. As the goal was to explore design space exploration, the data collection ended when a final concept had been chosen. Therefore, the two last stages $^{\mathrm{a}}$ of the design process in Figure 8 are empty. The results of this study suggests that using more than one design representation, particularly using physical models, leads to a broader exploration of the design space.

a. This paper used the same model as this thesis (see Figure 3) but the name used for the first stage was Ideation instead of Concept design. 


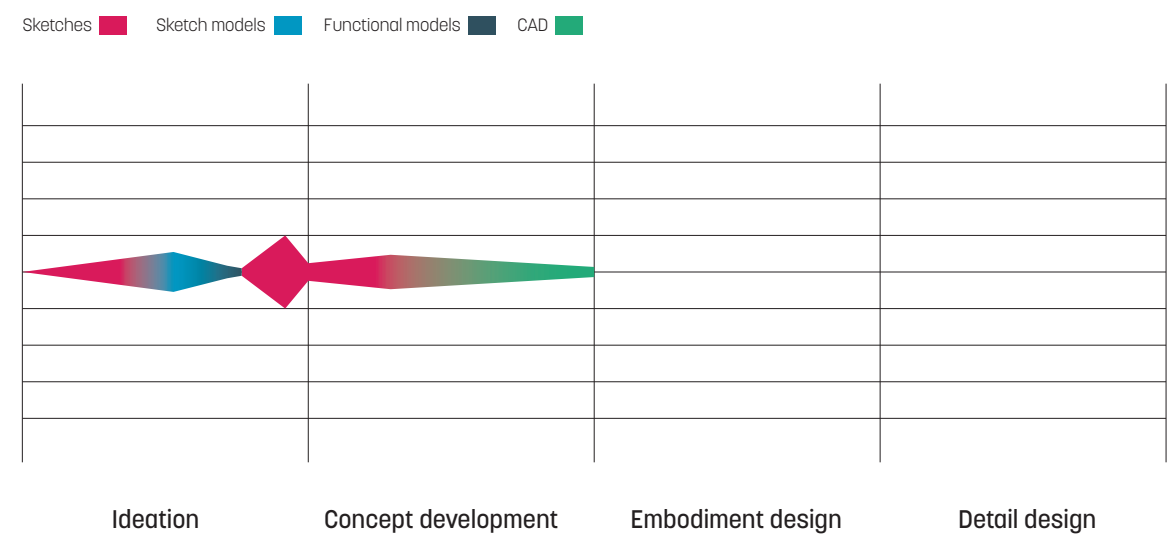

Figure $\mathbf{8}$ Example of a graph showing design representations used (colour) and diversity of ideas (height of graph)

\subsection{SHEDDING LIGHT ON GAME ENGINES AND VIRTUAL REALITY FOR AUTOMOTIVE LIGHTING DESIGN}

Paper $\mathrm{C}$ was a case study with the purpose to explore how game engines and VR can support design ideation in automotive lighting design. The two cases were master theses that explore using game engines and VR tools for design ideation in exterior and interior automotive lighting design. In the first case, the exterior lights of a car were visualized using a game engine (Figure 9) and evaluated using expert evaluation. In the second case, a game engine combined with VR was used to explore the ideation and concept development of interior lighting in trucks. The results from the two cases suggest that game engines, both with and without VR, can be used to support ideation in automotive lighting design. Furthermore, the insights from the case authors using game engines and VR for design ideation suggests that the tools have a use in design ideation outside the area of automotive lighting. 


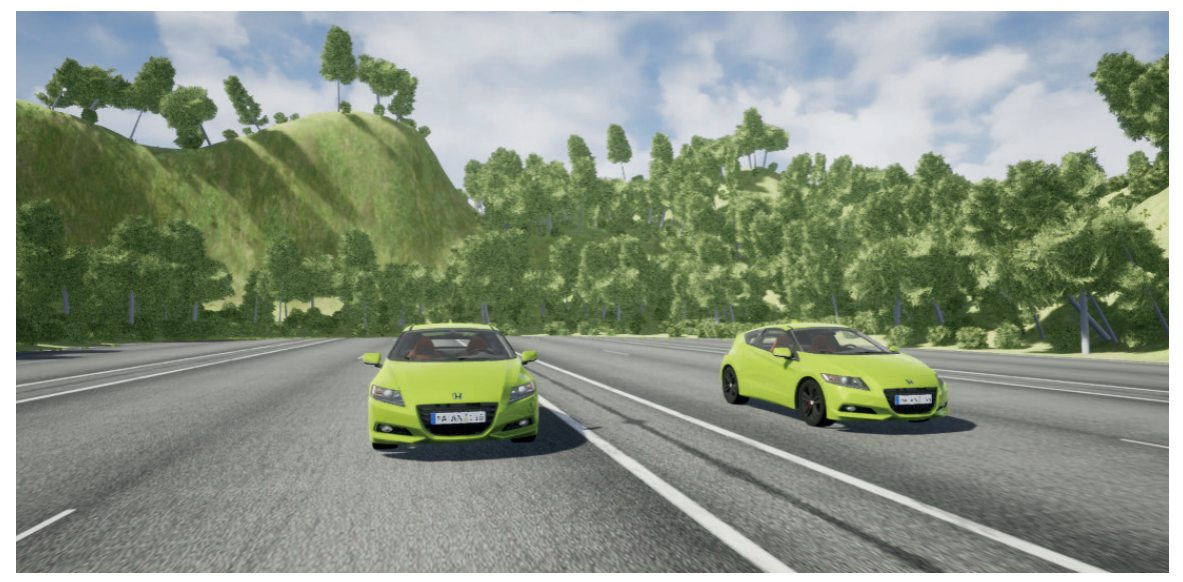

Figure 9 Car and environment visualization using Unreal Engine 4

\subsection{VIRTUAL REALITY SKETCHING}

Paper D explored the use of VR sketching in design ideation. It consisted of a pilot case and a pre-study for a controlled experiment (see Chapter 7). The case was part of a master thesis with the aim to design an orienteering shoe for a Swedish shoe company. The student recorded the use of the VR tool Gravity Sketch ("Gravity Sketch," 2017) during some of the ideation. This was used to gather initial data. The insights from the case were used to design a comparative study, comparing VR sketching to pen-and-paper sketching. A pre-study was undertaken with two groups of $5^{\text {th }}$ year Industrial design engineering students $(\mathrm{n}=16)$. They were asked to do two different ideation tasks. One group used sketching for both tasks and the other used sketching first and then the Gravity Sketch (Figure 10). The pen-and-paper sketches were collected after the task was done, while VR sketches were captured in a recording, as the final sketch will not show their workflow. The number of sketches and concepts per participant was counted and the workflow used when VR sketching was observed. The results from the pre-study suggest that the workflow used when VR sketching is different from pen-and-paper sketching, and that surfaces or volumes could be more effective for ideation in VR than 
line drawings. Moreover, it was observed that the students using VR sketching often chose to undo instead of keeping an imperfect shape or line. This way of undoing and re-drawing resulted in fewer completed sketches and opportunities for reinterpretations.

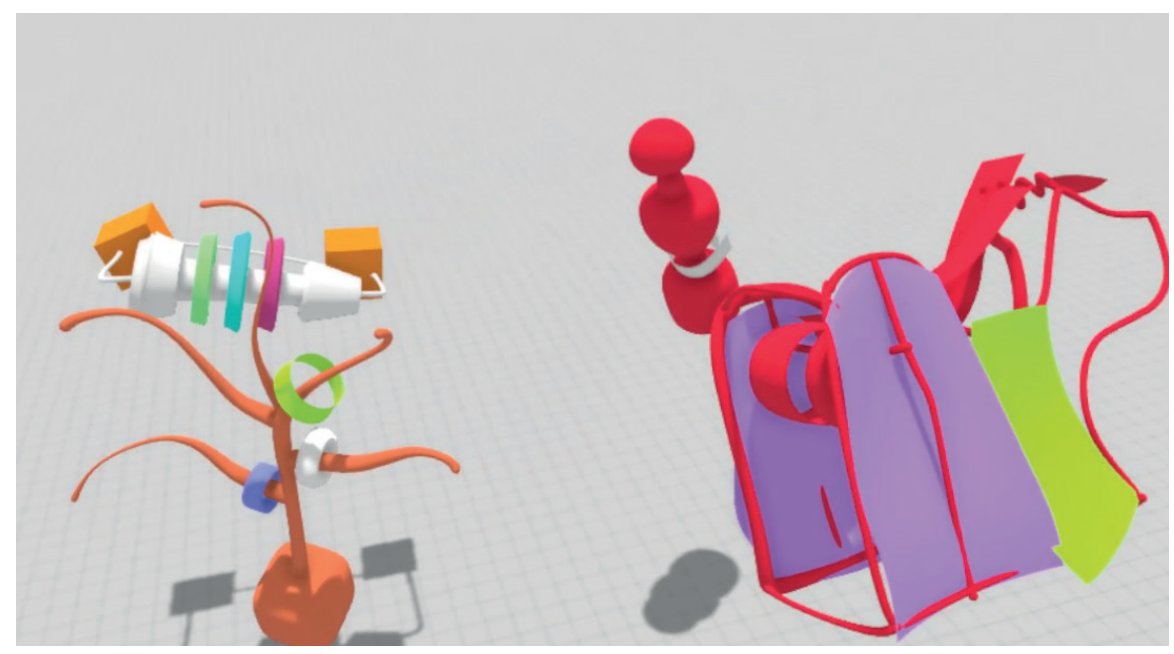

Figure 10 VR sketching a tape holder for multiple tapes in Gravity sketch 



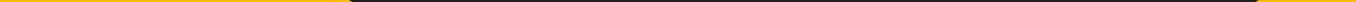

32 


\section{OUTCOMES}

In this chapter, the findings from the four studies are presented. The findings are sorted under two headings, each corresponding to one of the research questions.

The overarching result from the four studies is that there are several opportunities for the use of CAD tools in design ideation, which challenges the notion in literature that $\mathrm{CAD}$ tools have limited to no use for design ideation.

\subsection{WHAT POTENTIAL STRENGTHS DO CAD TOOLS HAVE WHEN EMPLOYED FOR DESIGN IDEATION?}

Below, the findings in regard to how CAD tools can benefit and support ideation are presented. Table 2 shows the benefits of CAD tools for design ideation that were identified in study A. Below that, more findings regarding how $\mathrm{CAD}$ tools can support design ideation are presented.

Table 2 Strengths of CAD tools for design ideation identified in study A

\begin{tabular}{l} 
Easy transformation [rotation, scaling, zooming and panning] (Pour \\
Rahimian et al., 2012; Ranscombe \& Bissett-Johnson, 2017) \\
\hline Ability to temporally omit an object or group of objects (Pour \\
Rahimian et al., 2012; Ranscombe \& Bissett-Johnson, 2017) \\
\hline Automatic undo and redo (Pour Rahimian et al., 2012; \\
Ranscombe \& Bissett-Johnson, 2017) \\
\hline Facilitates the creation of shapes that are hard to do manually \\
(Ranscombe \& Bissett-Johnson, 2017; Séquin, 2004) \\
Supports visualization of lighting (Andersson, 2017; Jönsson, 2017) \\
\hline Supports accurate representation of materials (Study A] \\
\hline Can be used to automatically generate design solutions (Study A] \\
\hline Supports design in a 1:1 scale when using VR (Study A]
\end{tabular}


ONE MORE TOOL IN THE TOOLBOX

The experts in study A argued that a benefit of using CAD tools for ideation is that they afford a different way of thinking about the problem. Thus, using CAD tools for design ideation will add another tool in the designers' toolbox, which will increase their ability to efficiently ideate on different kinds of problems.

\section{SUPPORTS IDEATION WHEN IT IS DIFFICULT TO SKETCH}

In some areas of design, such as lighting design, the objects being designed do not easily lend themselves to sketching. Here, CAD tools can be used to visualize what is hard to sketch and create a setting where reflective conversation and serendipitous interpretation is possible. While the suitability of a given CAD tool will vary depending on design problem and application area, the cases in Paper $\mathrm{C}$ showed that Game Engines and VR can be used to support ideation in automotive lighting design. In a previous study by Hjelseth, Morrison and Nordby (2015), it was suggested that game engines can be used to trigger reflective conversation, which was corroborated in Study C.

\section{ALLOWS DIFFERENT KINDS OF EXPERIMENTATION}

In the focus group discussion in Paper A, the experts argued that CAD tools allow for experimentation with ideas in ways that are not possible with sketching. Transform operations, such as scaling and deforming the object, are easy and instant in CAD tools (Pour Rahimian et al., 2012; Ranscombe \& Bissett-Johnson, 2017) which affords experimentation with sizing and transformation. In an example with a playground design, one of the experts recounted how a CAD tool allowed him to scale the whole model up or down and get an instant preview of how the playground would look in different sizes. In addition, using VR allows full scale representation of objects. This was a benefit of CAD tools suggested by one of the experts in Study A. It was briefly explored and found useful in the second case of study C, where the reachability of a light fixture was explored by using a full scale model of a truck cabin, lying down with the VR headset at the approximate height of the driver's bed and trying to reach it with the hand controller. 


\subsection{WHAT ARE THE EFFECTS OF USING CAD TOOLS FOR DESIGN IDEATION?}

How the use of a CAD tool will affect the design ideation will to a large extent depend on the tool, individual preference and skill and the particularities of the design task. However, there are certain common properties of CAD tools that affect the ideation process. These are expanded on below.

\section{DIFFERENT WORKFLOW}

It is obvious that the way of working, the workflow, when using a CAD tool is different from the workflow using pen-and-paper sketching. However, the studies in this thesis suggest that the notion that CAD tools cannot support reflective conversation, serendipitous interpretation and creativity is no longer true. However, getting started in a CAD tool still has a significantly higher threshold than pen-and paper-sketching. This is true for both the time and effort it takes to get started. The experts in study A argued that there are some CAD tools, such as SketchUp, that have a quite low threshold for getting started but that very few, if any, can match the ubiquitous and immediate nature of pen and paper.

\section{INSTANT UNDO}

The ability to instantly undo your actions is one of the fundamental differences between sketching and CAD tools. In Paper A, this was identified as one of the strengths of CAD tools. However, in Paper D, the ability to undo seems to have been more of a drawback, as the students using VR sketching were prone to undo and re-draw until they reached their intended result, which resulted in fewer completed sketches and fewer opportunities for reinterpretation. Thus, while being able to rapidly undo mistakes seems to be beneficial to the ideation process, achieving a faster workflow with less time redoing and more time working on creating, it might hinder reinterpretation and result in fewer ideas. 


\section{SINGLE TOOL PROCESS}

Using CAD tools for design ideation has long been envisioned as a way to streamline the process from initial idea to finished product by avoiding the time-consuming conversion to digital models when the ideation is finished. However, the findings from the studies suggest that this can have negative effects on ideation. In the master theses that were compared in study B, the students using more than one design representation showed a broader exploration of the design space. Moreover, the experts in study A argued that there is a benefit of using different tools because the switch between tools requires you to adopt a different way of thinking about the problem because of the different capabilities and workflows. 



\section{D) DISCUSSION \& IMPLICATIONS}

In this chapter, the contributions and implications of the findings from the four studies are highlighted and discussed in relation to literature. This is followed by a reflection on the research approach and methodology, including its limitations.

The main argument against CAD tools for design ideation in previous studies has been that CAD tools do not allow for reflective conversation and serendipitous interpretation. However, the studies in this thesis suggests that there can be reflective conversation (e.g. when using game engines, see Paper C) and serendipity (see Paper A) when using CAD tools in design ideation. By changing the view on using CAD tools in design ideation, this could enable new ways of working with ideation in design. In areas where it is currently difficult to use sketching to ideate, such as lighting design, using CAD tools could enable earlier exploration of different solutions and shorten the iteration time. Moreover, by presenting several examples of how CAD tools can be used for design ideation, this thesis presents a diverse view on CAD tools' different uses and capabilities that contests the misconception in literature that all CAD tools have the same characteristics (Lee \& Yan, 2016). Below the specific outcomes are discussed and compared with literature.

\subsection{UNDO OR UNDON'T}

One of the major differences between CAD tools and pen-and-paper sketching is the function to undo and redo actions instantly. However, it is unclear if this is a strength, as proposed by Ibrahim and Pour Rahimian (2010), or a weakness for design ideation. In support for the former view, Wojtczuk and Bonnardel (2011) found that CAD tool users had a higher number of corrected errors than manual (polystyrene block) modellers, which they interpreted as the undo feature making CAD tool users less hesitant about experimenting with 
different options. In contrast, the participants using VR sketching in Paper D were observed to undo and re-draw until they reached their intended result, which resulted in fewer completed sketches and fewer opportunities for reinterpretation. Thus, it seems that the ability to instantly undo can be either liberating or limiting. This suggests further studies exploring which of these is more prevalent and what can be done to mitigate the limiting effects of undoing in design ideation.

\subsection{FULL SCALE DESIGNING}

Having the ability to interact with full scale models in VR was proposed as a benefit of CAD tools by one of the experts in study A. It was explored briefly in case 2 of study $\mathrm{C}$ and found useful for experimenting with the reachability of a light fixture. Similarly, Jackson and Keefe (2016) found that viewing architectural models at life-size scale in VR can be beneficial for evaluation. They also note that correctly indicating the scale might be important during ideation and reflect that a feature that quickly toggles between life-size scale and a smaller working scale would be beneficial. Using VR for its full-scale capabilities has recently been reported in the automotive industry. McLaren Automotive use VR when comparing different interior designs as they claim it is superior to physical models often used for automotive design, offering the same sense of space but allowing much faster switching between concepts (Holmes, 2017). Moreover, in 2019 the automotive company Ford started employing VR sketching tool Gravity sketch ("Gravity Sketch," 2017), partly for its ability to design at full scale (Ford Motor company, 2019). Thus, based on the studies that have been undertaken and the recent use in practice, full scale design is suggested to be a useful benefit for design ideation. 


\subsection{WORKFLOW}

One of the main arguments against CAD tools for ideation has been that the workflow, that is proposed, including the interface (e.g. Kosmadoudi et al. (2013)), does not encourage experimentation, reinterpretation and serendipitous interpretation. However, the studies in this thesis suggest that this is no longer true. That being said, there are still parts of the workflow in CAD tools that can be negative for design ideation. The threshold for getting started in CAD tools is higher than pen-and-paper sketching and the skills required to use some CAD tools might not be part of designers' skill set. Case 1 in Study $\mathrm{C}$ showed that using game engines for design ideation on automotive lighting design requires more than basic programming skills, as adding realistic weather effects proved to be too difficult. Hjelseth, Morrison and Nordby (2015) reflected that a team containing both a designer and a programmer would be preferable, but that this might make the workflow too complicated for design work.

\subsection{SINGLE TOOL PROCESS}

While one of the goals of using CAD tools for design ideation have been to streamline the process by reducing the number of times the design needs to be remade or converted for a new tool, the studies in this thesis suggests that this might not be beneficial for design ideation. While a single tool process might be streamlined in terms of time, the results from study A and B suggests that a process where a single (CAD) tool is used for design ideation will reduce the breadth of design space exploration. Another effect of only using CAD tools for the whole design process is the loss of materiality, the tangible interaction with a physical artefact. The materiality of physical objects has been argued to be of importance for design ideation in several studies (e.g. Yang (2005) and Kelley (2001)). Häggman et al. (2015) found that designs made with physical models were perceived as having higher novelty than designs created using sketching or $\mathrm{CAD}$, which they link to rough models allowing a broader exploration 
of the design space. In the same study, they found that foam models were rated top for creativity and aesthetics while CAD correlated with low creativity. There are however efforts made to bring materiality to CAD tools, both by simulating materiality using haptic devices (e.g. (Liu, Dodds, McCartney, \& Hinds, 2004; Nagai, Tanoue, Akahane, \& Sato, 2015) and by integrating the physical reality and virtual world using Augmented Reality.

\subsection{ADDITIONS TO LITERATURE}

Previous studies comparing a CAD tool and pen-and-paper sketching generally only compare the CAD tool's ability to retain or emulate the strengths of sketches while ignoring the CAD specific benefits for design ideation that the tool might have. Therefore, an approach where both the strengths of sketches and CAD tools are taken into account is argued to be a fairer comparison. Thus, the evaluation method in Paper A, where CAD tools were rated on their ability to 1) retain the strengths of sketches and 2) add the strengths of CAD presents a novel approach to determine the use of CAD tools in design ideation.

The compilation of strengths and weaknesses of sketches and CAD that was required for the evaluation is an addition to literature on the use of CAD tools for design ideation. While some papers list strengths and/or weaknesses of sketches or CAD tools (e.g. (Buxton (2007) and Pour Rahimian et al. (2012)), no earlier research, to the authors knowledge, has compiled the strengths and weaknesses of sketches and CAD tools reported in literature. This can be used as a starting point for future research.

While game engines have been used for design applications before, especially in architecture and construction (e.g. Indraprastha \& Shinozaki (2009)), the use of a game engine for lighting design and the ideation of exterior and interior lighting designs are novel applications for game engines in design. 


\subsection{REFLECTIONS ON LIMITATIONS}

This thesis used an approach with four independent studies with each study exploring different CAD tools. The goal was to gather examples of situations where CAD tools can be useful for design ideation and explore the current practices of designers and design students so that the four studies could present several perspectives on the use of CAD tools for design ideation. While having four independent studies does not produce the same deep knowledge as a series of studies where each new study builds on the findings of the previous, it allows for a broader exploration which better served the purpose of this thesis.

As this research was primarily exploratory and the quantitative data comes from a limited samples, its limitations lies in that the results cannot be statistically proven or reliably generalized. However, as stated in the title, this was a first "sketch" of Computer Aided Ideation that aimed to explore the use of currently available CAD tools for design ideation. Thus, there are opportunities for future studies to validate the results from this thesis. Below follows a reflection on the four papers.

\section{PAPER A}

The compilation of strengths and weaknesses of CAD tools is limited to the strengths and weaknesses reported in literature and, as there are currently many different CAD tools, there are most likely strengths and weaknesses that are not included in this compilation. Moreover, they are based on literature from different design fields, time periods, and interpretations of what is and is not ideation. To improve the reliability, the experts were asked to review and expand the list. However, while the three experts are knowledgeable in their respective field, more design professionals, preferably from other design fields are needed to verify the strengths and weaknesses of CAD tools for design ideation. Lastly, when evaluating the tools, the experts were not given any input to guide their rating. Thus, to improve reliability, a common design problem, e.g. the design of a garden tool, could have been used to frame the evaluation. 


\section{PAPER B}

The previous master theses on product development which formed the dataset in this study were readily available at the start of the thesis work, which presented an opportunity for a study. The reason for choosing finished theses instead of studying master theses in progress and documenting their work was that the available theses provided a wider sample of works. However, this means that the results are dependent on what the students chose to show in their theses, which is a limitation. The rationale for this method was based on the assumption that the students show their best work in their theses and that this is representative of their breadth of exploration. The choice of method came with the inherent limitation that there was no opportunity to ask the students about their work. Thus, when it was unclear what in the thesis was an idea and what was not, this had to be decided by the researcher.

\section{PAPER C}

Study $\mathrm{C}$ followed two cases in the area of automotive lighting design. As with the study B, this study used secondary data in the form of two master theses. However, in this case, the authors of the theses were involved in the study, which increases reliability. As both cases were undertaken in the area of lighting design, this increases the reliability of the conclusions for this specific application area.

\section{PAPER D}

This study consisted of two parts, one case and a comparative experiment with 16 participants. The case was used to gather initial data on VR sketching and resulted in several interesting insights. However, VR sketching was only used during a part of the design ideation, meaning that the insights are preliminary. The main insight, that VR sketching is used more as a modelling tool than a sketching tool, was however not evident in the experiment. The comparative experiment was undertaken as a pre-study for a later study, implying a preliminary study set-up. While the number of ideas and concepts were counted and compared, the most interesting insight was how the ability to quickly and easily undo in VR made the participants undo and re-draw until they reached a satisfactory result. 



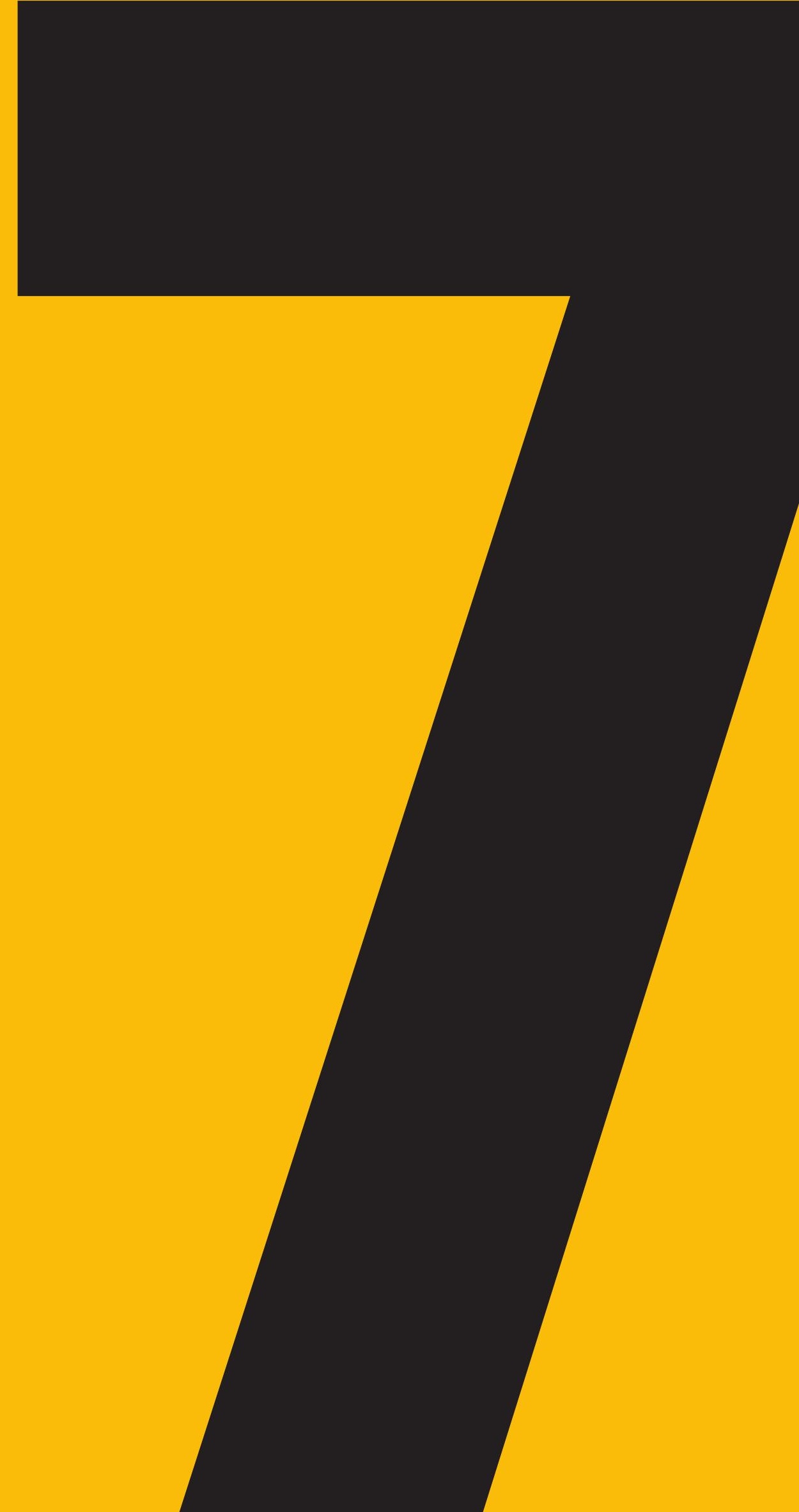




\section{D) CONCLUSIONS \& FURTHER STUDIES}

This chapter presents the conclusions of this licentiate thesis. Overall, the results of the studies in this thesis suggests that the previously held notion that CAD tools are not useful for design ideation is no longer true. Based on expert evaluations and case studies, this thesis concludes that there are several opportunities for the use of CAD tools in design ideation. This is certainly true in design fields where it is difficult to make sketches, such as lighting design. However, the results do not indicate that currently available CAD tools should replace penand-paper sketching. Sketching has a long history in design and will most likely not be replaced within the foreseeable future. Instead the results of the studies suggest that CAD tools can be used as an alternative or complement to sketching in design ideation. This can provide more possibilities for the use of CAD tools in design ideation and may lead to new ways of working with ideation in design.

\section{RQ1: WHAT POTENTIAL STRENGTHS DO CAD TOOLS HAVE WHEN EMPLOYED} FOR DESIGN IDEATION?

Using CAD tools for ideation affords a different way of thinking about the problem. Thus, using CAD tools for ideation will add another tool in the designers' toolbox, which will increase the ability to efficiently ideate on different kinds of problems. Computer Aided Design tools have the ability to display $3 \mathrm{D}$ representation of $3 \mathrm{D}$ object, which in combination with VR allows the representation and design of objects in full scale. With their ability to visualize things that are hard to sketch, CAD tools be used for design ideation in areas where it is hard to make sketches. Also, the ability to perform instantaneous transform operations, such as scaling and deforming, affords experimentation with sizing and transformation. 
RQ2: WHAT ARE THE EFFECTS OF USING CAD TOOLS FOR DESIGN IDEATION?

Every CAD tool affords a different workflow. What they have in common is that this workflow is different from the traditional way of working with pen-and-paper sketches, but the notion that the workflow in CAD tools does not encourage experimentation, reinterpretation and serendipitous interpretation is no longer true. However, getting started in a CAD tool still has a significantly higher threshold than pen and paper. The ability to instantly undo in CAD tools has been identified as both a potential strength and a potential weakness for design ideation. While being able to instantly undo mistakes could be beneficial to the ideation process, achieving a faster workflow with less time redoing and more time working on creating, this might also result in fewer opportunities for reinterpretation. Future studies will have to determine which one is more prevalent and what can be done to mitigate the limiting effects of undoing in design ideation.

\subsection{TENTATIVE FUTURE WORK}

This thesis has focused on exploring the field of CAD tools for design ideation. From this, several new areas of inquiry have been discovered, such as how the ability to instantly undo and the ability to design in a full scale (in VR) affects the ideation process. These insights, particularly how undoing affects the ideation process, will tentatively be investigated using controlled experiments in combination with retrospective protocol analysis. In addition to this, the author will continue to explore the use of CAD tools for design ideation from different perspectives, tentatively using research through design methodology (Stappers \& Giaccardi, 2017) and reflecting on the use of a CAD tool for design ideation in a manner of reflective practice (Schön, 1991). 


\subsection{FURTHER STUDIES}

\section{COLLABORATIVE DESIGN IDEATION}

While the design process is very much a team activity, with a lot of collaboration, most CAD tools of today are made for a single user. For design ideation, using pen-and-paper sketching to allow several designers to sketch simultaneously has great value (van Der Lugt, 2005), enabling a parallel ideation workflow and increasing the opportunities for reinterpretation. However, pen-and-paper sketching is most effective when the participants are in the same location (Ibrahim \& Pour Rahimian, 2010). As projects are getting increasingly globalized with designers from different locations involved, there are opportunities for using $\mathrm{CAD}$ tools to enable remote collaborative ideation.

\section{GENERATIVE DESIGN}

Generative design was highlighted as one of the strengths of CAD tools by the experts in study A. The area of generative design, letting computers generate output that meet certain criteria such as weight and stiffness, is very much in its early stage. However, the ability of CAD tools to generate shapes that are hard for humans to imagine and even harder to create could be very useful in ideation. This will however change the role of designer from creator to decision maker and the tool will become active and human initiated instead of passive and human controlled. While this could be very beneficial for ideation, there might be a problem of decision fatigue (or ego depletion, see (Baumeister, 2002)), when choosing between many similar solutions. Moreover, when the computer generates solutions there might not be any happy or unhappy accidents, as it will only respond to the constraints set by the designer. 


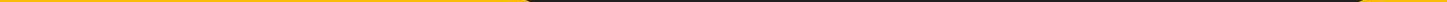




\section{川 REFERENCES}

Alcaide-Marzal, J., Diego-Más, J. A., Asensio-Cuesta, S., \& Piqueras-Fiszman, B. (2013). An exploratory study on the use of digital sculpting in conceptual product design. Design Studies, 34(2), 264-284. https://doi.org/10.1016/j.destud.2012.09.001

Andersson, P. (2017). Exterior lighting design in the automotive industry. Linköping University.

Babapour, M. (2015). Pensieri - An inquiry into Sketching and Modelling in Design.

Baumeister, R. F. (2002). Ego Depletion and Self-Control Failure: An Energy Model of the Self's Executive Function. Self and Identity, 1(2), 129-136. https://doi.org/10.1080/152988602317319302

Bilda, Z., \& Demirkan, H. (2003). An insight on designers' sketching activities in traditional versus digital media. Design Studies, 24(1), 27-50. https://doi.org/10.1016/S0142-694X(02)00032-7

Bilda, Z., Gero, J. S., \& Purcell, T. (2006). To sketch or not to sketch? That is the question. Design Studies, 27(5), 587-613. https://doi.org/10.1016/j.destud.2006.02.002

Buxton, B. (2007). Sketching user experiences.

Charlesworth, C. (2007). Student Use of Virtual and Physical Modelling in Design Development - an Experiment in 3D Design Education, 10(1).

Chester, I. (2007). Teaching for CAD expertise. International Journal of Technology and Design Education, 17(1), 23-35. https://doi.org/10.1007/s10798-006-9015-z 
Clemens, A. (2018). When the Mind's Eye Is Blind.

Creswell, J. W. (2003). Research Design Qualitative, Quantitative. and Mixed Methods Approaches second edition sage Sage Publications International Educational and Professional Publisher Thousand Oaks London New Delhi. Retrieved from https://ucalgary. ca/paed/files/paed/2003_creswell_a-framework-for-design.pdf

Do, E. Y. L. (2005). Design sketches and sketch design tools. In Knowledge-Based Systems (Vol. 18, pp. 383405). https://doi.org/10.1016/j.knosys.2005.07.001

Dorst, K. (2003). The Problem of Design Problems. Design Thinking Research Symposium 6, 4(Creativity and Cognition Studio Press), 135-147. https://doi.org/10.1504/JDR.2004.009841

Dorta, T, Lesage, A., \& Perez, E. (2009). Design tools and collaborative ideation. Joining Languages, Cultures and Visions: CAADFutures, $65^{\circ}$ V79.

Dorta, Tomás, Pérez, E., \& Lesage, A. (2008). The ideation gap:. hybrid tools, design flow and practice. Design Studies, 29(2), 121-141. https://doi.org/10.1016/j.destud.2007.12.004

Ericsson, A. K., \& Simon, H. A. (1984). Protocol analysis: Verbal reports as data. MIT Press.

Ferguson, E. S. (1994). Engineering and the Mind's Eye. MIT press.

Fish, J. C. (1996). How sketches work: a cognitive theory for improved system design. Loughborough University of Technology.

Fish, J., \& Scrivener, S. (2007). Amplifying the Mind's

Eye : Sketching and Visual Cognition, 23(1), 117-126. 
Ford Motor company. (2019). Ford collaboration with gravity sketch introduces co-creation feature, allowing designers across globe to work in same virtual reality space. Retrieved October 25, 2019, from http://www.campaign.ford.com/content/fordmedia/fna/us/en/ news/2019/05/06/ford-collaboration-gravity-sketch-co-creation.html

Gero, J. S., \& Tang, H. H. (2001). The differences between retrospective and concurrent protocols in revealing the processoriented aspects of the design process. Design Studies, 22(3), 283-295. https://doi.org/10.1016/S0142-694X(00)00030-2

Gharib, I. (2013). Integration of Sketch-based Ideation and 3D Modeling with CAD Systems.

Goel, V. (1995). Sketches of thought. MIt Press.

Goldschmidt, G. (1991). The dialectics of sketching. Creativity Research Journal, 4(2), 123-143. https:// doi.org/10.1080/10400419109534381

Gravity Sketch. (2017). Retrieved September 13, 2017, from https://www.gravitysketch.com/

Hacker, W., Römer, A., Lindemann, U., Weißhahn, G., \& Pache, M. (2002). Effort-saving product representations in design-results of a questionnaire survey. Design Studies, 22(6), 473-491. https://doi.org/10.1016/s0142-694x(01)00003-5

Hanington, B., \& Martin, B. (2012). Universal methods of design: 100 ways to research complex problems, develop innovative ideas, and design effective solutions. Rockport Publishers.

Hanna, R., \& Barber, T. (2001). An inquiry into computers in design: Attitudes before-attitudes after. Design Studies, 22(3), 255-281. https://doi.org/10.1016/S0142-694X(00)00029-6 
Hennessey, J. M., \& Stappers, P. J. (1999). Computersupported tools for the conceptualization phase. Proceedings of 4th Int. Conf. on Design Thinking. MIT, 177-187.

Hjelseth, S., Morrison, A., \& Nordby, K. (2015). Design and Computer Simulated User Scenarios: Exploring RealTime 3D Game Engines and Simulation in the Maritime Sector. International Journal of Design, 9(3), 63-75.

Holmes, S. (2017). Everything is for a reason. Retrieved December 10, 2018, from https://www.develop3d.com/features/everything-isfor-a-reason-McLaren-virtual-reality-VR-automotive-design-CAD

Ibrahim, R., \& Pour Rahimian, F. (2010). Comparison of CAD and manual sketching tools for teaching architectural design. Automation in Construction, 19(8), 978-987. https://doi.org/10.1016/j.autcon.2010.09.003

Indraprastha, A., \& Shinozaki, M. (2009). The Investigation on Using Unity3D Game Engine in Urban Design Study. ITB Journal of Information and Communication Technology, 3(1), 1-18. https://doi.org/10.5614/itbj.ict.2009.3.1.1

Jackson, B., \& Keefe, D. F. (2016). Lift-Off: Using Reference Imagery and Freehand Sketching to Create 3D Models in VR. IEEE Transactions on Visualization and Computer Graphics, 22(4), 1442-1451. https://doi.org/10.1109/TVCG.2016.2518099

Jonson, B. (2002). Sketching now. International Journal of Art and Design Education, 21(3), 246253. https://doi.org/10.1111/1468-5949.00321

Jonson, B. (2005). Design ideation: The conceptual sketch in the digital age. Design Studies, 26(6), 613624. https://doi.org/10.1016/j.destud.2005.03.001 
Jönsson, J. (2017). Virtual concept development of interior lighting in the automotive industry. Linköping University.

Kelley, T. (2001). Prototyping is the Shorthand of Design of innovation. Design Management Journal, 12(3), 35-42.

Knight, M., Dokonal, W., Brown, A., \& Hannibal, C. (2005). Contemporary Digital Techniques in the Early Stages of Design. Computer Aided Architectural Design Futures 2005, 165-174. https://doi.org/10.1007/1-4020-3698-1_15

Kosmadoudi, Z., Lim, T., Ritchie, J., Louchart, S., Liu, Y., \& Sung, R. (2013). Engineering design using game-enhanced CAD: The potential to augment the user experience with game elements. CAD Computer Aided Design, 45(3), 777-795. https://doi.org/10.1016/j.cad.2012.08.001

Lee, S., \& Yan, J. (2016). The impact of 3D CAD interfaces on user ideation: A comparative analysis using SketchUp and Silhouette Modeler. Design Studies, 44, 52-73. https://doi.org/10.1016/j.destud.2016.02.001

Lim, S., Qin, S. F., Prieto, P., Wright, D., \& Shackleton, J. (2004). A study of sketching behaviour to support free-form surface modelling from on-line sketching. Design Studies, 25(4), 393-413. https://doi.org/10.1016/j.destud.2003.10.008

Liu, X., Dodds, G., McCartney, J., \& Hinds, B. K. (2004). Virtual DesignWorks - Designing 3D CAD models via haptic interaction. CAD Computer Aided Design, 36(12), 1129-1140. https://doi.org/10.1016/j.cad.2003.10.003

Madsen, D. A., \& Madsen, D. P. (2016). Engineering Drawing and Design (sixth). Cengage Learning. 
Mothersill, P., Bove, V. M., \& Bove Jr, V. M. (2017).

Humans, Machines and the Design Process. Exploring the Role of Computation in the Early Phases of Creation. The Design Journal, 20(sup1), S3899-S3913. https:// doi.org/10.1080/14606925.2017.1352892

Musta'amal, A. H., Norman, E., \& Hodgson, T. (2008). CAD as a 'Recording' or 'Designing' Tool: Evidence From User Behaviours. In The Design and Technology Association International Research Conference 2008, [Loughborough University, 2-4 July]. Wellesbourne: The Design and Technology Association (pp. 47-54).

Musta'amal, A. H., Norman, E., \& Hodgson, T. (2009). Gathering Empirical Evidence Concerning Links Between Computer Aided Design ( CAD ) and Creativity. Design and Technology ..., 14(2), 53-66.

Musta'amal, A., Norman, E., \& Hodgson, T. (2009). Observing Creative Behaviours. The Design and Technology Association International Research Conference 2009, 60-67.

Nagai, K., Tanoue, S., Akahane, K., \& Sato, M. (2015).

Wearable 6-DoF wrist haptic device "SPIDAR-W," 1-2. https://doi.org/10.1145/2818384.2818403

Oh, J., Stuerzlinger, W., \& Danahy, J. (2005). Comparing sesame and sketching on paper for conceptual $3 \mathrm{~d}$ design. EG Workshop on Sketch-Based ..., 81-88. https://doi.org/10.2312/SBM/SBM05/081-087

Pei, E. (2009). Building a Common Language of Design Representations for Industrial Designers \& Engineering Designers, 658. 
Pei, E., Campbell, I., \& Evans, M. A. (2009). Building a Common Ground - The Use of Design Representation Cards for Enhancing Collaboration between Industrial Designers and Engineering Designers. Design Research Society Conference, 18. Retrieved from http://shura.shu.ac.uk/450/

Pour Rahimian, F., Ibrahim, R., \& Jaafar, M. F. Z. (2012). A Feasibility Study for Developing 3D Sketching Concept in Virtual Reality (VR) Environment. ALAM CIPTA, International Journal on Sustainable Tropical Design Research \& Practice, 3(1), 69-79.

Purcell, A. T., \& Gero, J. S. (1998). Drawings and the Design Process. Design Studies, 19(4), 389-430. https:// doi.org/10.1016/S0142-694X(98)00015-5

Ranscombe, C. C., \& Bissett-Johnson, K. (2017). Digital Sketch Modelling: Integrating digital sketching as a transition between sketching and CAD in Industrial Design Education. Design and Technology Education, 22(1), 1-15. https://doi.org/10.21606/drs.2016.69

Robertson, B. F., \& Radcliffe, D. F. (2009). Impact of CAD tools on creative problem solving in engineering design. CAD Computer Aided Design, 41(3), 136-146. https://doi.org/10.1016/j.cad.2008.06.007

Schön, D. (1991). The reflective practitioner: How professionals think in action. Ashgate Publishing Limited.

Sener, B., Vergeest, J. S. M., \& Akar, E. (2002). New Generation Computer-Aided Design Tools : Two Related Research Projects Investigating the Future Expectations of Designers. International Design Conference, 539-544.

Séquin, C. H. (2004). CAD Tools for Aesthetic Engineering. Computer-Aided Design and Applications, 1(1-4), 301-309. https://doi.org/10.1080/16864360.2004.10738271 
Shih, Y. T., Sher, W. D., \& Taylor, M. (2017). Using suitable design media appropriately: Understanding how designers interact with sketching and CAD modelling in design processes. Design Studies, 53, 47-77. https://doi.org/10.1016/j.destud.2017.06.005

Simon, H. A. (1970). The Sciences of the Artificial. Technology and Culture (Vol. 11). https://doi.org/10.2307/3102825

Simon, H. A. (1973). The structure of ill structured problems. Artificial Intelligence, 4(3-4), 181-201. https://doi.org/10.1016/0004-3702(73)90011-8

Stappers, P. J., \& Giaccardi, E. (2017). Research through Design. In Soegaard, M. \& Friis-Dam, R. (eds.),The Encyclopedia of HumanComputer Interaction, 2nd edition. Retrieved from http://www. interaction-design.org/literature/book/the-encyclopedia-ofhuman-computer-interaction-2nd-ed/research-through-design

Stappers, P. J., \& Hennessey, J. M. (1999). Toward Electronic Napkins and Beermats: Computer Support for Visual Ideation Skills. Visual Representations and Interpretations, 220-225. https://doi.org/10.1007/978-1-4471-0563-3_24

Stones, C., \& Cassidy, T. (2007). Comparing synthesis strategies of novice graphic designers using digital and traditional design tools. Design Studies, 28(1), 59-72. https://doi.org/10.1016/j.destud.2006.09.001

Stones, C., \& Cassidy, T. (2010). Seeing and discovering: How do student designers reinterpret sketches and digital marks during graphic design ideation? Design Studies, 31(5), 439-460. https://doi.org/10.1016/j.destud.2010.05.003

Sutherland, I. E. (1968). A head-mounted three dimensional display. Proceedings of the December 9-11, 1968, Fall Joint Computer Conference, Part I on - AFIPS '68 (Fall, Part I), 757. https://doi.org/10.1145/1476589.1476686 
Suwa, M., \& Tversky, B. (1997). What Do Architects and Students Perceive in their Design Sketches? A Protocol Analysis. Design Studies, 18(4), 385-403. https://doi.org/ http://dx.doi.org/10.1016/S0142-694X(97)00008-2

Teegavarapu, S., Summers, J. D., \& Mocko, G. M. (2008). Case study method for design research: A justification. Proceedings of the ASME Design Engineering Technical Conference, 4(January 2008), 495-503. https://doi.org/10.1115/DETC2008-49980

Thomas Buser. (n.d.). History of Drawing. Retrieved May 26, 2019, from http://historyofdrawing.com/

Tovey, M., Porter, S., \& Newman, R. (2003). Sketching, concept development and automotive design. Design Studies, 24(2), 135-153. https://doi.org/10.1016/S0142-694X(02)00035-2

Ulrich, K. T., \& Eppinger, S. D. (2008). Product design and development (Fourth). New York: MacGraw-Hill.

van Boeijen, A., Daalhuizen, J., Zijlstra, J., \& Van der Schoor, R. (2014). Delft design guide: Design strategies and methods. BIS publishers.

van Der Lugt, R. (2005). How sketching can affect the idea generation process in design group meetings. Design Studies, 26(2), 101-112. https://doi.org/10.1016/j.destud.2004.08.003

van Dijk, C. G. C. C. (1995). New insights in computeraided conceptual design. Design Studies, 16(1), 62-80. https://doi.org/10.1016/0142-694X(95)90647-X

Verstijnen, I. M., Van Leeuwen, C., Goldschmidt, G., Hamel, R., \& Hennessey, J. M. (1998). Sketching and creative discovery. Design Studies, 19(4), 519-546. https://doi.org/10.1016/S0142-694X(98)00017-9 
Vries, E. De. (2019). Problem solving in design : A comparison of three theories, 9003(1990).

Wikberg-Nilsson, Å., Ericson, Å., \& Törlind, P. (2015). Design: process och metod. (1st ed.). Lund: Studentlitteratur.

Wizarding world. (2017). Retrieved October 17, 2019, from https://www.wizardingworld.com/ features/the-hardest-wizarding-world-spells

Wojtczuk, A., \& Bonnardel, N. (2011). Designing and assessing everyday objects: Impact of externalisation tools and judges' backgrounds. Interacting with Computers, 23(4), 337-345. https://doi.org/10.1016/j.intcom.2011.05.004

Won, P. H. (2001). The comparison between visual thinking using computer and conventional media in the concept generation stages of design. Automation in Construction, 10(3), 319-325. https://doi.org/10.1016/S0926-5805(00)00048-0

Yang, M. C. (2005). A study of prototypes, design activity, and design outcome. Design Studies, 26(6), 649-669. https://doi.org/10.1016/j.destud.2005.04.005 
Varma mackor är gott...med pålägg.

Valgot Steensland 
Below, the contributions of the authors is outlined.

\section{PAPER A}

This study was conceived and performed by the thesis author. The paper was written by the thesis author with feedback from the main supervisor (Wever). Paper title by Wever.

\section{PAPER B}

The idea of studying finished master theses came from Wever. The study was performed by the thesis author. The paper was written by the thesis author with feedback from Wever. Paper title by Wever.

\section{PAPER C}

The master theses used in this study were written by Patrik Andersson and Johan Jönsson, who were both supervised by Wever. The idea of using these as cases for a study came from Wever. Analysis of the cases and literature review was performed by the thesis author, with feedback from Wever. Paper title by the thesis author.

\section{PAPER D}

This study was conceived and performed by the thesis author. The case used in the study was a part of the master thesis by Jens Wängdahl who was supervised by Wever. However, it was the thesis author who asked Wängdahl to use VR-sketching as a part of his ideation process. The paper was written by the thesis author with feedback from Wever. Paper title by the thesis author.

\section{Papers}

\section{The papers associated with this thesis have been removed for copyright reasons. For more details about these see:}

http://urn.kb.se/resolve?urn=urn:nbn:se:liu:diva-162022 


\section{DEPARTMENT OF SCIENCE AND TECHNOLOGY}

Linköping Studies in Science and Technology No. 1859 [2019]

Division of Machine Design

Department of Management and Engineering

Linköping University

SE-601 74 Linköping, Sweden

www.liu.se
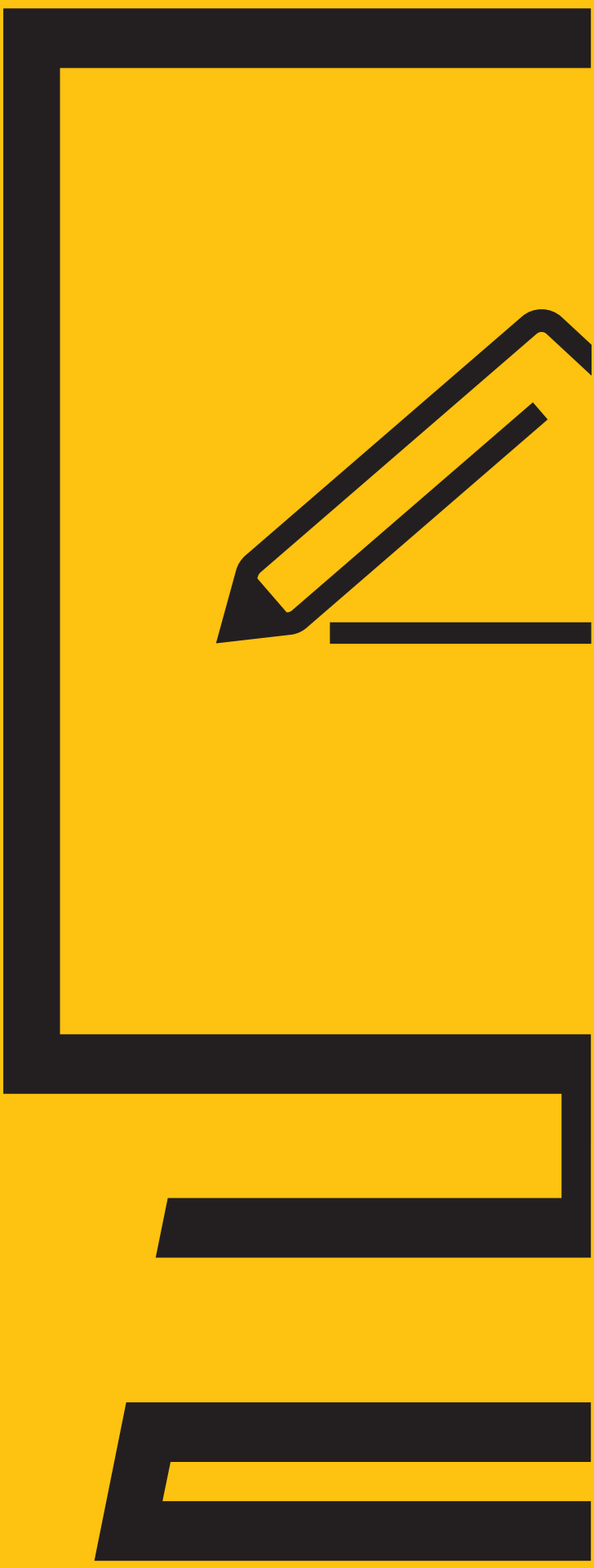